Publ. RIMS, Kyoto Univ.

32 (1996), 277-322

\title{
Binormal Deformation and Bimicrolocalization
}

By

\author{
Kiyoshi TAKeUCHI*
}

\section{Contents}

$\S 1$. Introduction

§2. Geometry

2.1. Preliminary results on normal bundles

2.2. Binormal deformation

2.3. Binormal cones

$\S 3 . \quad$ Bispecialization Functor

3.1. Functorial properties of the functor $v_{N M}$

3.2. Structure of the functor $v_{N M}$

$\S 4$. Bimicrolocalization Functors

4.1. Functors $v \mu_{N M}$ and $\mu_{N M}$

4.2. Functorial properties of $\nu \mu_{N M}$ and $\mu_{N M}$

4.3. Various generalizations of the functor $\mu$ hom

§5. Applications

5.1. Second microfunctions associated to a submanifold

5.2. Second microfunctions associated to a foliation

5.3. Second microdifferential operators

\section{§ 1. Introduction}

The specialization functor is an important tool in algebraic geometry as well as in algebraic analysis. In the real case, it associates to a sheaf $F$ on a real manifold $X$ and to a submanifold $M$ of $X$, a sheaf (i.e. an object of the derived category of sheaves) $v_{M}(F)$ on the normal bundle $T_{M} X$ which describes the "boundary values" of $F$ along $M$. Its Fourier transform is the sheaf $\mu_{M}(F)$ of Sato's microlocalization of $F$ along $M$.

Communicated by M. Kashiwara, March 29, 1995.

1991 Mathematics Subject Classification(s): 14F05, 32C38, 35A27, 58 G07.

* Department of Mathematics, Hiroshima University, 1-3-1, Kagamiyama, Higashi-Hiroshimashi, 739, Hiroshima, Japan. 
Now consider a triplet of real manifolds $N \subset M \subset X$. In order to analyze the sheaf $v_{M}(F)$ along the submanifold $N \times{ }_{M} T_{M} X$ of $T_{M} X$, a natural idea is to introduce the second specialization $v_{\left(N \times_{M} T_{M} X\right)}\left(v_{M}(F)\right)$. Taking the Fourier transform of this object, we get the theory of the second microlocalization introduced by M. Kashiwara [4] in 1972 and developed by many authors, in particular by Kashiwara-Kawaï [7] and Laurent [15]. However, the sheaf one obtains by this method is very "big" and the direct image of this sheaf on the bundle $N \times{ }_{M} T_{M}^{*} X$ is not the restriction to $N \times{ }_{M} T_{M}^{*} X$ of the sheaf $\mu_{M}(F)$. This leads to many difficulties.

In this paper, we propose a new approach to the second specialization by considering the inverse image $\tilde{M}$ of $M$ in $\tilde{X}_{N}$, the normal deformation of $X$ along $N$ and defining the binormal deformation of $X$ along $(M, N)$ as the deformation of $\tilde{X}_{N}$ along $\tilde{M}$. Then, by paraphrasing the construction of the functor $v_{M}(\cdot)$, we construct the functor $v_{N M}(\cdot)$ which associates to a sheaf $F$ on $X$ a sheaf on $T_{N} M \times{ }_{M} T_{M} X$. By taking the Fourier transform of this sheaf, we obtain the sheaf $v \mu_{N M}(F)$ on $T_{N} M \times{ }_{M} T_{M}^{*} X$ and the sheaf $\mu_{N M}(F)$ on $T_{N}^{*} M \times{ }_{M} T_{M}^{*} X$. We study the functorial properties of these new sheaves and we show that they enjoy the desired properties. In particular the direct image of $\mu_{N M}(F)$ on $N \times{ }_{M} T_{M}^{*} X$ coincides with the restriction of $\mu_{M}(F)$ to this set.

It would then be possible to develop a whole theory of the functor $\mu_{N M}(\cdot)$, in the lines of Sato-Kawai-Kashiwara [19] or Kashiwara-Schapira [11], but this will not be done here. Let us only mention that the author ([24] and [25]) has applied this theory to the study of boundary value problems in higher codimension.

Finally, notice that the idea of second normal deformation already appeared in Sabbah [18] (this idea has been developed by Bost [1] to obtain a proof of Atiyah-Singer theorem) and a construction of a sheaf of second microfunctions, following the lines of [19], based on a geometrical idea very closed to ours, had been proposed by Kataoka-Tose in [13]. We thank $\mathbf{N}$. Tose to have drawn our attention on this (unpublished) paper. Another construction (based on integral formulas) of the same sheaf of second microfunctions was performed later in [14]. Also notice that Sjöstrand [23] introduced a different language and with different methods, various spaces of second microfunctions. On the other hand, "simultaneous specialization" is a slightly different geometrical construction introduced by Delort [2].

The main results of this paper were announced in [22]. The idea of bispecialization is essentially due to P. Schapira. The author and he tried to realize his idea to construct a framework of higher codimensional boundary value problems treated in [24] and [25]. In this process, the relation with the theory of second microlocal analysis was found and developed by the author. The present article gives complete proofs of this theory including the 
developments after the introduction of bispecialization. The author would like to express his profound gratitude to Prof. Schapira for giving him many valuable suggestions and technical aids throughout this work. This theory were not realized without his constant encouragements. $\mathrm{He}$ is also very grateful to- Prof. Honda and Mr. Watanabe for careful reading of some parts of this manuscript.

\section{§ 2. Geometry}

\subsection{Preliminary results on normal bundles}

Let $X \supset M \supset N$ be a sequence of $C^{\infty}$-manifolds. Let us denote it by $(X, M, N)$ and call it a triplet of manifolds. We sometimes identify $M$ with the zero-section of the normal bundle $T_{M} X$, and $N$ is considered as a submanifold of $T_{M} X$. We recall some elementary geometrical results on normal bundles.

Proposition 2.1. Let $(X, M, N)$ be a triplet of manifolds. There are canonical isomorphisms of normal bundles:

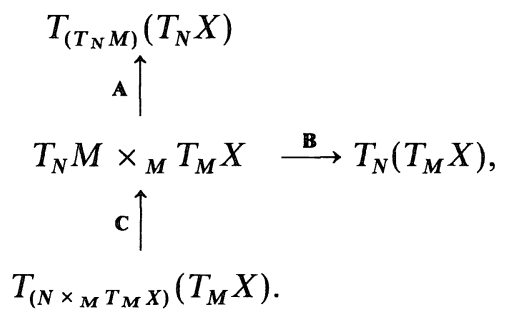

where $N$ is considered as a submanifold of the zero section $M$ of $T_{M} X$ and $T_{N} M$ is imbedded into $T_{N} X$ by the tangent map associated to the inclusion $(M, N) \rightarrow(X, N)$.

Proof. We will construct the canonical morphisms A, B and C. Then, using local coordinate systems, it will be obvious that these morphisms are isomorphisms. For this purpose, the simple lemma below is crucial.

Lemma 2.2. Let $\tau: E \rightarrow Z, \quad(x ; \xi) \mapsto x$ be a vector bundle over $a$ $C^{\infty}$-manifold $Z$.

(i) Let $\left(x, \xi ; x^{*} \frac{\partial}{\partial x}+\xi^{*} \frac{\partial}{\partial \xi}\right)$ be a linear coordinate system on TE. Then, there exists a canonical injective morphism of vector bundles over $Z$ :

$$
\psi_{\tau}: E \longrightarrow Z \times_{E} T E, \quad(x, \xi) \longmapsto\left(x, 0 ; \xi \frac{\partial}{\partial \xi}\right) .
$$


(ii) Let $E_{1}$ and $E_{2}$ be two copies of $E$ and $\left(\left(x, \xi_{1}\right),\left(x, \xi_{2}\right)\right)$ be a linear coordinate system on $E_{1} \times{ }_{Z} E_{2}$. Then there exists a canonical injective morphism of vector bundles over $E_{1}$ :

$$
\phi_{\tau}: E_{1} \times{ }_{Z} E_{2} \longrightarrow T E_{1}, \quad\left(\left(x, \xi_{1}\right),\left(x, \xi_{2}\right)\right) \longmapsto\left(x, \xi_{1} ; \xi_{2} \frac{\partial}{\partial \xi_{1}}\right) .
$$

Proof.

(i) First consider the exact sequence:

$$
0 \longrightarrow T Z \longrightarrow Z \times_{E} T E \longrightarrow T_{Z} E \longrightarrow 0 .
$$

This sequence splits since the projection $E \rightarrow Z$ gives the morphism $T E \rightarrow E \times_{Z}$ $T Z$, hence the morphism: $Z \times_{E} T E \rightarrow T Z$. Therefore we get the injection:

$$
\psi_{\tau}: E \simeq T_{Z} E \longrightarrow Z \times_{E} T E .
$$

(ii) Applying (i) to the vector bundle $E_{1} \times{ }_{Z} E_{2} \rightarrow E_{1}$, we get the injective morphism :

$$
E_{1} \times{ }_{Z} E_{2} \longrightarrow E_{1} \times{ }_{\left(E_{1} \times{ }_{Z} E_{2}\right)} T\left(E_{1} \times{ }_{Z} E_{2}\right) \longrightarrow T\left(E_{1} \times{ }_{Z} E_{2}\right) .
$$

Next consider the tangent map: $T\left(E_{1} \times{ }_{Z} E_{2}\right) \rightarrow T E_{1}$ associated to the addition map $E_{1} \times{ }_{Z} E_{2} \rightarrow E_{1}$. The composition of these two morphisms is the desired one :

$$
\phi_{\tau}: E_{1} \times{ }_{Z} E_{2} \longrightarrow T E_{1} .
$$

Applying Lemma 2.2 (i) to $\tau: T_{M} X \rightarrow M$, we have an injective morphism of vector bundles over $M$ :

$$
\psi_{\tau}: T_{M} X \longrightarrow M \times_{\left(T_{M} X\right)} T\left(T_{M} X\right) .
$$

Restricting it to $N$, we get a chain of morphisms on $N$ :

$$
N \times_{M} T_{M} X \underset{\psi_{\tau} \mid N}{\longrightarrow} N \times_{\left(T_{M} X\right)} T\left(T_{M} X\right) \longrightarrow T_{N}\left(T_{M} X\right)
$$

The composition of the above morphisms gives a morphism:

$$
N \underset{M}{\times} T_{M} X \longrightarrow T_{N}\left(T_{M} X\right)
$$

On the other hand, we have a tangent map associated to the zero section embedding $(M, N) \rightarrow\left(T_{M} X, N\right)$, that is :

$$
T_{N} M \longrightarrow T_{N}\left(T_{M} X\right) .
$$


By adding the morphisms (2.6) and (2.7), we get the morphism $\mathbf{B}$ :

$$
\text { B: } T_{N} M \times{ }_{M} T_{M} X \longrightarrow T_{N}\left(T_{M} X\right) .
$$

To construct the morphism A, first notice the commutative diagram:

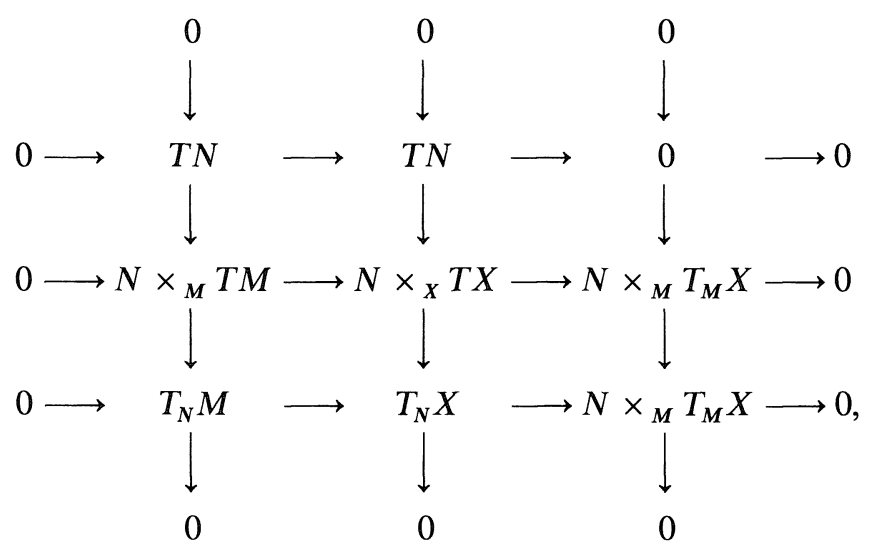

where all columns and the first and the second rows are exact. Hence, by virtue of the nine Lemma, the third row is also exact. That is, we have a canonical isomorphism of vector bundles over $N$ :

$$
T_{N} X / T_{N} M \stackrel{\sim}{\longrightarrow} N \times_{M} T_{M} X
$$

in which the left hand side is a quotient bundle over $N$.

If we apply Lemma 2.2 (ii) to $\tau_{1}: T_{N} M \rightarrow N$ and $\tau_{2}: T_{N} X \rightarrow N$, we get:

$$
\phi_{\tau_{1}}: T_{N} M \times{ }_{N} T_{N} M \longrightarrow T\left(T_{N} M\right)
$$

and

$$
\phi_{\tau_{2}}: T_{N} X \times{ }_{N} T_{N} X \longrightarrow T\left(T_{N} X\right)
$$

Since $\phi_{\tau_{2}}$ is a morphism of vector bundles over $T_{N} X$, it can be restricted to $T_{N} M \subset T_{N} X$, and we denote it by $\left.\phi_{\tau_{2}}\right|_{T_{N} M}$. Now we have a commutative diagram:

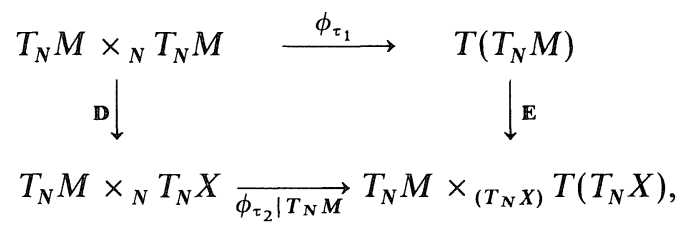

where the morphism $\mathrm{D}$ is induced by the injection $T_{N} M \rightarrow T_{N} X$, and the morphism $\mathbb{E}$ is the tangent map associated to the inclusion $T_{N} M \hookrightarrow T_{N} X$. The 
commutativity is easily checked by fixing a local coordinate system. Therefore, we can construct a morphism between the quotient bundle of the morphism $\mathbf{D}$ and that of $\mathbf{E}$ :

$$
T_{N} M \times{ }_{N}\left(T_{N} X / T_{N} M\right) \longrightarrow T_{\left(T_{N} M\right)}\left(T_{N} X\right) .
$$

The left hand side is isomorphic to $T_{N} M \times_{M} T_{M} X$ by (2.10), and we have thus constructed the morphism $\mathrm{A}$.

The morphism $\mathbf{C}$ is the tangent map induced by the projection:

$$
\left(T_{M} X, N \times{ }_{M} T_{M} X\right) \longrightarrow(M, N),
$$

that is,

$$
T_{\left(N \times{ }_{M} T_{M} X\right)}\left(T_{M} X\right) \longrightarrow\left(N \times{ }_{M} T_{M} X\right) \times{ }_{N} T_{N} M \simeq T_{N} M \times{ }_{M} T_{M} X .
$$

This completes the proof of Proposition 2.1.

\subsection{Binormal deformation}

Let $(X, M, N)$ be a triplet of manifolds. First, recall the construction of the normal deformation of $X$ along $N$ in [11]. We shall denote it by $\tilde{X}_{N}$ and let $t_{1} \in \mathbb{R}$ be the deformation parameter. Then we have the commutative diagram below:

$$
\begin{array}{ll}
T_{N} X \stackrel{s_{N}}{\longrightarrow} & \tilde{X}_{N} \stackrel{j_{N}}{\stackrel{1}{j_{N}}} \Omega_{N}=\left\{t_{1}>0\right\} \\
\tau_{N} \downarrow & p_{N} \downarrow
\end{array}
$$

Set $\hat{\Omega}_{N}:=\left\{\left(x, t_{1}\right) \in \tilde{X}_{N} ; t_{1} \neq 0\right\}$ and consider :

$$
\tilde{M}:=\overline{\left(p_{N} \mid \hat{\Omega}_{N}\right)^{-1} M} \subset \tilde{X}_{N},
$$

the closure of $\left(p_{N} \mid \hat{\Omega}_{N}\right)^{-1} M$ in $\tilde{X}_{N}$. Then $\tilde{M}$ is an intrinsically defined smooth submanifold of $\tilde{X}_{N}$. If we choose a local coordinate system $x=\left(x^{\prime}, x^{\prime \prime}, x^{\prime \prime \prime}\right)$ of $X$ such that

$$
\left[\begin{array}{l}
M=\left\{x^{\prime}=0\right\}, \\
N=\left\{x^{\prime}=0, x^{\prime \prime}=0\right\},
\end{array}\right.
$$

then $\tilde{M}=\left\{x^{\prime}=0\right\}$ in $\tilde{X}_{N}$. Now we shall define the binormal deformation $\tilde{X}_{N M}$ of $X$ along $(M, N)$ as the normal deformation of $\tilde{X}_{N}$ along $\tilde{M}$.

Definition 2.3. One sets :

$$
\tilde{X}_{N M}:=\left(\tilde{X}_{N}\right) \tilde{\tilde{M}} .
$$

Define the morphism $p: \tilde{X}_{N M} \rightarrow X$ as the composition of the natural morphisms 
$p_{\tilde{M}}: \tilde{X}_{N M} \rightarrow \tilde{X}_{N}$ and $p_{N}: \tilde{X}_{N} \rightarrow X$. Using the local coordinate system in (2.18), this morphism $p$ is described as follows:

$$
\left(x^{\prime}, x^{\prime \prime}, x^{\prime \prime \prime}, t_{1}, t_{2}\right) \longmapsto\left(t_{2} x^{\prime}, x^{\prime \prime}, x^{\prime \prime \prime}, t_{1}\right) \longmapsto\left(t_{1} t_{2} x^{\prime}, t_{1} x^{\prime \prime}, x^{\prime \prime \prime}\right),
$$

where $t_{2}$ denotes the deformation parameter of $\tilde{X}_{N M} \rightarrow \tilde{X}_{N}$. Since $\tilde{M} \cap\left\{t_{1}=0\right\}$ $\simeq T_{N} M \subset T_{N} X$ in $\tilde{X}_{N}$, we have an isomorphism:

$$
\tilde{X}_{N M} \cap\left\{t_{1}=0\right\} \simeq\left(\tilde{X}_{N} \cap\left\{t_{1}=0\right\}\right){\tilde{\left(\tilde{M} \cap\left\{t_{1}=0\right\}\right)}}_{\left(T_{N} X\right)_{\left(T_{N} M\right)}^{\tilde{x}_{N}}}
$$

and

$$
\tilde{X}_{N M} \cap\left\{t_{1}=t_{2}=0\right\} \simeq T_{\left(T_{N} M\right)}\left(T_{N} X\right) .
$$

By virtue of Proposition 2.1, we can identify it with $T_{N} M \times{ }_{M} T_{M} X$, and there exists a commutative diagram below:

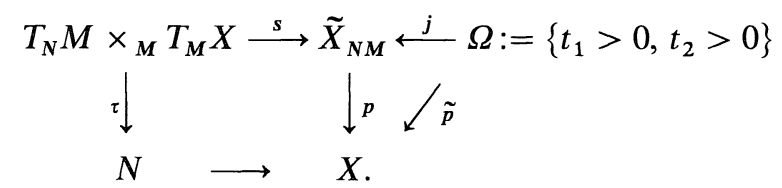

Remark 2.4. (i) $\tilde{M}$ is isomorphic to the normal deformation $\tilde{M}_{N}$ of $M$ along $N$.

(ii) We have another construction of $\tilde{X}_{N M}$ starting from the normal deformation $p_{M}: \tilde{X}_{M} \rightarrow X$ with the deformation parameter $t_{2}$. Setting:

$$
\tilde{N}:=\overline{\left(\left.p_{M}\right|_{\left\{t_{2} \neq 0\right\}}\right)^{-1} N} \subset \tilde{X}_{M}
$$

the binormal deformation $\tilde{X}_{N M}$ of $X$ along $(M, N)$ can be defined by:

$$
\tilde{X}_{N M}:=\left(\tilde{X}_{M}\right) \tilde{\tilde{N}} \text {. }
$$

Of course, it is also possible to construct $\tilde{X}_{N M}$ directly by gluing local charts as in [11].

\subsection{Binormal cones}

There is a $\left(\mathbb{R}^{+}\right)^{2}$-action

$$
\tilde{X}_{N M} \times\left(\mathbb{R}^{+}\right)^{2} \longrightarrow \tilde{X}_{N M}
$$

on $\tilde{X}_{N M}$ which is described in the local coordinate system $\left(x^{\prime}, x^{\prime \prime}, x^{\prime \prime \prime}, t_{1}, t_{2}\right)$ associated to the one in (2.18) of $X$ by:

$$
\left(\left(x^{\prime}, x^{\prime \prime}, x^{\prime \prime \prime}, t_{1}, t_{2}\right),(c, d)\right) \longmapsto\left(c d x^{\prime}, c x^{\prime \prime}, x^{\prime \prime \prime}, \frac{t_{1}}{c}, \frac{t_{2}}{d}\right) .
$$

It is easily checked that this definition is intrinsic. 
Definition 2.5. Let $S$ be a subset of $X$. We define the binormal cone to $S$ along $(M, N)$ by:

$$
C_{N M}(S):=\left(T_{N} M \times{ }_{M} T_{M} X\right) \cap \overline{\tilde{p}^{-1}(S)} .
$$

This is a closed subset of $T_{N} M \times{ }_{M} T_{M} X$.

In this section, we will investigate the properties of $C_{N M}(S)$. The problems are always local, and we shall restrict ourselves to the following situation:

$$
\left\{\begin{array}{l}
X=X_{x^{\prime}}^{\prime} \times X_{x^{\prime \prime}}^{\prime \prime} \times X_{x^{\prime \prime \prime}}^{\prime \prime \prime} \\
M=\{0\} \times X_{x^{\prime \prime}}^{\prime \prime} \times X_{x^{\prime \prime \prime}}^{\prime \prime \prime} \\
N=\{0\} \times\{0\} \times X_{x^{\prime \prime \prime}}^{\prime \prime \prime},
\end{array}\right.
$$

where $X_{x^{\prime}}^{\prime}=\mathbb{R}_{x^{\prime}}^{n^{\prime}}, X_{x^{\prime \prime}}^{\prime \prime}=\mathbb{R}_{x^{\prime \prime}}^{n^{\prime \prime}}$ and $X^{\prime \prime \prime}=\mathbb{R}_{x^{\prime \prime \prime}}^{n^{\prime \prime \prime}}$ for some $n^{\prime}, n^{\prime \prime}$ and $n^{\prime \prime \prime}$. Then there exists an associated coordinate system $\left(v^{\prime} \frac{\partial}{\partial x^{\prime}}, v^{\prime \prime} \frac{\partial}{\partial x^{\prime \prime}}, x^{\prime \prime \prime}\right)$ of

$$
T_{N} M \times{ }_{M} T_{M} X \simeq \mathbb{R}_{v^{\prime}}^{n^{\prime}} \times \mathbb{R}_{v^{\prime \prime}}^{n^{\prime \prime}} \times \mathbb{R}_{x^{\prime \prime \prime}}^{n^{\prime \prime \prime}}
$$

Proposition 2.6. Let $S$ be a subset of $X$.

(i) $C_{N M}(S)$ is a closed biconic subset of $T_{N} M \times{ }_{M} T_{M} X$.

(ii) $\left(v_{0}^{\prime}, v_{0}^{\prime \prime}, x_{0}^{\prime \prime \prime}\right) \in C_{N M}(S) \Leftrightarrow$ There exists a sequence $\left\{\left(x_{n}^{\prime}, x_{n}^{\prime \prime}, x_{n}^{\prime \prime \prime}\right), c_{n}, d_{n}\right\}$ in $S \times\left(\mathbb{R}^{+}\right)^{2}$ with $x_{n}=\left(x_{n}^{\prime}, x_{n}^{\prime \prime}, x_{n}^{\prime \prime \prime}\right)$ such that

$$
\left\{\begin{array}{l}
x_{n} \longrightarrow\left(0,0, x_{0}^{\prime \prime \prime}\right), \\
c_{n} d_{n} x_{n}^{\prime} \longrightarrow v_{0}^{\prime}, \\
c_{n} x_{n}^{\prime \prime} \longrightarrow v_{0}^{\prime \prime} \\
d_{n} \longrightarrow+\infty
\end{array}\right.
$$

Proof.

(i) Since the restriction of the $\left(\mathbb{R}^{+}\right)^{2}$-action (2.25) to

$$
\tilde{X}_{N M} \cap\left\{t_{1}=t_{2}=0\right\}=T_{N} M \times{ }_{M} T_{M} X
$$

coincides with the biconic structure of $T_{N} M \times{ }_{M} T_{M} X$, the set $C_{N M}(S)$ is biconic.

(ii) (A) First assume $\left(v_{0}^{\prime}, v_{0}^{\prime \prime}, x_{0}^{\prime \prime \prime}\right) \in C_{N M}(S)$. Then there exists a sequence $\left\{x_{n}^{\prime}, x_{n}^{\prime \prime}, x_{n}^{\prime \prime \prime}, t_{n}, s_{n}\right\}$ in $\tilde{p}^{-1}(S)$ which satisfies:

$$
\left(x_{n}^{\prime}, x_{n}^{\prime \prime}, x_{n}^{\prime \prime \prime}, t_{n}, s_{n}\right) \longrightarrow\left(v_{0}^{\prime}, v_{0}^{\prime \prime}, x_{0}^{\prime \prime \prime}, 0,0\right) .
$$

We can easily see that the sequence $\left\{\left(t_{n} s_{n} x_{n}^{\prime}, t_{n} x_{n}^{\prime \prime}, x_{n}^{\prime \prime \prime}\right), \frac{1}{t_{n}}, \frac{1}{s_{n}}\right\}$ in $S \times\left(\mathbb{R}^{+}\right)^{2}$ satisfies the condition (2.30).

(B) Conversely, assume the condition (2.30). 
(a) The case when $\left\{c_{n}\right\}$ is not bounded.

Taking a subsequence if necessary, we may assume $c_{n} \rightarrow+\infty$. Then, the sequence in $\tilde{p}^{-1}(S)$ :

$$
\left\{\left(c_{n} d_{n} x_{n}^{\prime}, c_{n} x_{n}^{\prime \prime}, x_{n}^{\prime \prime \prime}\right), \frac{1}{c_{n}}, \frac{1}{d_{n}}\right\}
$$

converges to $\left(v_{0}^{\prime}, v_{0}^{\prime \prime}, x_{0}^{\prime \prime \prime}, 0,0\right) \in T_{N} M \times_{M} T_{M} X$. Thus, we have $\left(v_{0}^{\prime}, v_{0}^{\prime \prime}, x_{0}^{\prime \prime \prime}\right) \in$ $C_{N M}(S)$.

(b) The case when $\left\{c_{n}\right\}$ and $\left\{c_{n} d_{n}\right\}$ are bounded. Because $x_{n}=\left(x_{n}^{\prime}, x_{n}^{\prime \prime}\right.$, $\left.x_{n}^{\prime \prime \prime}\right) \rightarrow\left(0,0, x_{0}^{\prime \prime \prime}\right)$,

$$
\left\{\begin{array} { l } 
{ c _ { n } d _ { n } x _ { n } ^ { \prime } \longrightarrow 0 } \\
{ c _ { n } x _ { n } ^ { \prime \prime } \longrightarrow 0 }
\end{array} \text { and } \left\{\begin{array}{l}
v_{0}^{\prime}=0 \\
v_{0}^{\prime \prime}=0
\end{array}\right.\right.
$$

Moreover, taking a subsequence, we may assume

$$
\left|x_{n}^{\prime}\right|<\frac{1}{4^{n}} \quad \text { and } \quad\left|x_{n}^{\prime \prime}\right|<\frac{1}{2^{n}} \quad \text { for } \forall n \in \mathbb{N} .
$$

If we define the sequences $\left\{\varepsilon_{n}\right\}$ and $\left\{\delta_{n}\right\}$ by:

$$
\varepsilon_{n}=\frac{n}{2^{n}}, \delta_{n}=\frac{1}{2^{n}}
$$

then we have:

$$
\left(\left(\frac{x_{n}^{\prime}}{\varepsilon_{n} \delta_{n}}, \frac{x_{n}^{\prime \prime}}{\varepsilon_{n}}, x_{n}^{\prime \prime \prime}\right), \varepsilon_{n}, \delta_{n}\right) \longrightarrow\left(\left(0,0, x_{0}^{\prime \prime \prime}\right), 0,0\right) \in C_{N M}(S) .
$$

(c) The case when $\left\{c_{n}\right\}$ is bounded and $\left\{c_{n} d_{n}\right\}$ is not bounded. Taking a subsequence, it may be assumed:

$$
\left\{\begin{array}{l}
\left|x_{n}^{\prime \prime}\right|<\frac{1}{n^{2}} \\
\left|\frac{1}{c_{n} d_{n}}\right|<\frac{1}{n^{2}},
\end{array}\right.
$$

because $x_{n}^{\prime \prime} \rightarrow 0$. If we set $\varepsilon_{n}:=\frac{1}{n}$, we have:

$$
\left(c_{n} d_{n} x_{n}^{\prime}, \frac{x_{n}^{\prime \prime}}{\varepsilon_{n}}, x_{n}^{\prime \prime \prime}, \varepsilon_{n}, \frac{1}{\varepsilon_{n} c_{n} d_{n}}\right) \in \tilde{p}^{-1}(S)
$$

and it converges to the point: 


$$
\left(v_{0}^{\prime}, v_{0}^{\prime \prime}, x_{0}^{\prime \prime \prime}, 0,0\right)=\left(v_{0}^{\prime}, 0, x_{0}^{\prime \prime \prime}, 0,0\right) \in T_{N} M \times{ }_{M} T_{M} X .
$$

Therefore this completes the proof of Proposition 2.6.

There is another interpretation of $C_{N M}(S)$.

Proposition 2.7. Let $p_{0}=\left(v_{0}^{\prime}, v_{0}^{\prime \prime}, 0\right) \in T_{N} M \times_{M} T_{M} X$ and set:

$$
\Gamma_{\varepsilon}:=\left\{\left(x^{\prime}, x^{\prime \prime}, x^{\prime \prime \prime}\right) \in X ;\left|x^{\prime}\right|<\varepsilon\left|x^{\prime \prime}\right|\right\} .
$$

(i) Assume $v_{0}^{\prime} \neq 0, v_{0}^{\prime \prime} \neq 0$. Then:

$$
p_{0} \notin C_{N M}(S)
$$

$$
\Longleftrightarrow\left\{\begin{array}{l}
\text { there exist an open neighborhood } U \text { of } 0, \\
\text { open convex cones } \Gamma^{\prime} \subset X_{x^{\prime}}^{\prime} \text { and } \Gamma^{\prime \prime} \subset X_{x^{\prime \prime}}^{\prime \prime} \\
\text { which satisfy } v_{0}^{\prime} \in \Gamma^{\prime} \text { and } v_{0}^{\prime \prime} \in \Gamma^{\prime \prime} \text { respectively, } \\
\text { and } \varepsilon>0, \text { such that: } \\
S \cap\left\{U \cap\left(\Gamma^{\prime} \times \Gamma^{\prime \prime} \times X_{x^{\prime \prime \prime}}^{\prime \prime \prime}\right) \cap \Gamma_{\varepsilon}\right\}=\varnothing,
\end{array}\right.
$$

(ii) Assume $v_{0}^{\prime}=0, v_{0}^{\prime \prime} \neq 0$. Then:

$$
\begin{aligned}
p_{0}= & \left(0, v_{0}^{\prime \prime}, 0\right) \notin C_{N M}(S) \\
& \Longleftrightarrow \begin{array}{l}
\text { there exist an open neighborhood } U \text { of } 0, \\
\text { an open convex cone } \Gamma^{\prime \prime} \subset X_{x^{\prime \prime}}^{\prime \prime} \text { which satisfy } \\
v_{0}^{\prime \prime} \in \Gamma^{\prime \prime}, \text { and } \varepsilon>0, \text { such that: } \\
S \cap\left\{U \cap\left(X_{x^{\prime}}^{\prime} \times \Gamma^{\prime \prime} \times X_{x^{\prime \prime \prime}}^{\prime \prime \prime}\right) \cap \Gamma_{\varepsilon}\right\}=\emptyset
\end{array}
\end{aligned}
$$

(iii) Assume $v_{0}^{\prime} \neq 0, v_{0}^{\prime \prime}=0$. Then:

$$
\begin{aligned}
p_{0}= & \left(v_{0}^{\prime}, 0,0\right) \notin C_{N M}(S) \\
& \Longleftrightarrow \begin{array}{l}
\text { there exist an open neighborhood } U \text { of } 0 \text { and } \\
\text { an open convex cone } \Gamma^{\prime} \subset X_{x^{\prime}}^{\prime} \text { which satisfies } \\
v_{0}^{\prime} \in \Gamma^{\prime}, \text { such that: } \\
S \cap\left\{U \cap\left(\Gamma^{\prime} \times X_{x^{\prime \prime}}^{\prime \prime} \times X_{x^{\prime \prime \prime}}^{\prime \prime \prime}\right)\right\}=\emptyset .
\end{array}
\end{aligned}
$$

(iv) Finally assume $v_{0}^{\prime}=0, v_{0}^{\prime \prime}=0$. Then:

$$
\begin{aligned}
p_{0}= & (0,0,0) \notin C_{N M}(S) \\
& \Longleftrightarrow\left\{\begin{array}{l}
\text { there exists an open neighborhood } U \text { of } 0, \\
\text { such that: } \\
S \cap U=\varnothing .
\end{array}\right.
\end{aligned}
$$

Proof. The parts $(\Leftarrow)$ are always easy, so we only prove the parts $(\Rightarrow)$. Throughout the proof, we use the results of the preceding proposition 
as a definition of $C_{N M}(S)$.

(i) Let $\left\{U_{n}\right\}_{n \in \mathbb{N}}$ be a decreasing sequence of open neighborhoods of 0 in $X$ such that:

$$
\bigcap_{n \in \mathbb{N}} U_{n}=\{0\}
$$

Let $\left\{\Gamma_{n}^{\prime}\right\}_{n \in \mathbb{N}}$ and $\left\{\Gamma_{n}^{\prime \prime}\right\}_{n \in \mathbb{N}}$ be decreasing sequences of open convex cones of $X_{x^{\prime}}^{\prime}$ and $X_{x^{\prime \prime}}^{\prime \prime}$ respectively which satisfy:

$$
\bigcap_{n \in \mathbb{N}} \Gamma_{n}^{\prime}=\left\{c \cdot v_{0}^{\prime} ; c \in \mathbb{R}^{+}\right\}, \bigcap_{n \in \mathbb{N}} \Gamma_{n}^{\prime \prime}=\left\{c \cdot v_{0}^{\prime \prime} ; c \in \mathbb{R}^{+}\right\} .
$$

We assume: for every $n \in \mathbb{N}$ there exists :

$$
x_{n}=\left(x_{n}^{\prime}, x_{n}^{\prime \prime}, x_{n}^{\prime \prime \prime}\right) \in S \cap\left\{U_{n} \cap\left(\Gamma_{n}^{\prime} \times \Gamma_{n}^{\prime \prime} \times X_{x^{\prime \prime \prime}}^{\prime \prime \prime}\right) \cap \Gamma_{\frac{1}{n}}\right) .
$$

Then, we have:

$$
\left\{\begin{array}{l}
x_{n} \longrightarrow 0, x_{n}^{\prime} \in \Gamma_{n}^{\prime}, x_{n}^{\prime \prime} \in \Gamma_{n}^{\prime \prime} \quad \text { and } \\
\left|x_{n}^{\prime}\right|<\frac{1}{n}\left|x_{n}^{\prime \prime}\right|, \quad \text { for every } n \in \mathbb{N}
\end{array}\right.
$$

Since the "directions" of $x_{n}^{\prime}$ and $x_{n}^{\prime \prime}$ tend to $v_{0}^{\prime}$ and $v_{0}^{\prime \prime}$ respectively, if we set

$$
d_{n}:=\frac{\left|v_{0}^{\prime}\right|}{\left|v_{0}^{\prime \prime}\right|} \cdot \frac{\left|x_{n}^{\prime \prime}\right|}{\left|x_{n}^{\prime}\right|}>\frac{\left|v_{0}^{\prime}\right|}{\left|v_{0}^{\prime \prime}\right|} \cdot n>0
$$

the "direction" of the vector $\left(\begin{array}{c}d_{n} x_{n}^{\prime} \\ x_{n}^{\prime \prime}\end{array}\right)$ tends to the vector $\left(\begin{array}{c}v_{0}^{\prime} \\ v_{0}^{\prime \prime}\end{array}\right)$, that is, there exists a sequence $c_{n}>0$, such that:

$$
c_{n}\left(\begin{array}{c}
d_{n} x_{n}^{\prime} \\
x_{n}^{\prime \prime}
\end{array}\right) \longrightarrow\left(\begin{array}{c}
v_{0}^{\prime} \\
v_{0}^{\prime \prime}
\end{array}\right)
$$

Moreover we have $d_{n} \rightarrow+\infty$ by (2.37). This contradicts the assumption that $\left(v_{0}^{\prime}, v_{0}^{\prime \prime}, 0\right) \notin C_{N M}(S)$.

(ii) Assume as in the proof of (i): for every $n \in \mathbb{N}$, there exists:

$$
x_{n} \in S \cap\left\{U_{n} \cap\left(X_{x^{\prime}}^{\prime} \times \Gamma_{n}^{\prime \prime} \times X_{x^{\prime \prime \prime}}^{\prime \prime \prime}\right) \cap \Gamma_{\frac{1}{n}}\right\} .
$$

First of all, since the "direction" of $x_{n}^{\prime \prime}$ tends to $v_{0}^{\prime \prime}$, there exists a sequence $c_{n}>0$, such that:

$$
c_{n} x_{n}^{\prime \prime} \longrightarrow v_{0}^{\prime \prime} \neq 0
$$


In particular, the sequence $\left\{c_{n} x_{n}^{\prime \prime}\right\}$ is bounded, and

$$
\left|c_{n} x_{n}^{\prime}\right|<\frac{1}{n}\left|c_{n} x_{n}^{\prime \prime}\right|<\frac{c}{n} \text { for some } c>0 .
$$

Now set $d_{n}:=\sqrt{n}>0$. Then:

$$
\left\{\begin{array}{l}
d_{n} \longrightarrow+\infty \\
c_{n} d_{n} x_{n}^{\prime} \longrightarrow 0 \\
c_{n} x_{n}^{\prime \prime} \longrightarrow v_{0}^{\prime \prime} \neq 0
\end{array}\right.
$$

This is a contradiction.

(iii) Assume: for every $n \in \mathbb{N}$, there exists :

$$
x_{n} \in S \cap\left\{U_{n} \cap\left(\Gamma_{n}^{\prime} \times X_{x^{\prime \prime}}^{\prime \prime} \times X_{x^{\prime \prime \prime}}^{\prime \prime \prime}\right)\right\} .
$$

Then, extracting a subsequence, we may assume:

$$
\left|x_{n}^{\prime}\right|<\frac{1}{n}, \text { for every } n \in \mathbb{N} .
$$

Since the "direction" of $x_{n}^{\prime}$ tends to $v_{0}^{\prime}$, by setting

$$
d_{n}:=\frac{\left|v_{0}^{\prime}\right|}{\left|x_{n}^{\prime}\right|}>\left|v_{0}^{\prime}\right| \cdot n>0,
$$

we have:

$$
d_{n} x_{n}^{\prime} \longrightarrow v_{0}^{\prime}
$$

Now set $c_{n} \equiv 1$ for every $n \in \mathbb{N}$, then we have a convergence:

This is a contradiction.

$$
\left\{\begin{array}{l}
d_{n} \longrightarrow+\infty \\
c_{n} d_{n} x_{n}^{\prime} \longrightarrow v_{0}^{\prime} \neq 0 \\
c_{n} x_{n}^{\prime \prime} \longrightarrow 0
\end{array}\right.
$$

(iv) Assume: there exists a sequence $x_{n} \in S$, such that $x_{n} \rightarrow 0$. Extracting a subsequence, we may assume that:

$$
\left|x_{n}^{\prime}\right|<\frac{1}{n}, \text { for every } n \in \mathbb{N} .
$$

Set $c_{n} \equiv 1, d_{n}:=\sqrt{n}$. Then :

$$
\left\{\begin{array}{l}
d_{n} \longrightarrow+\infty \\
c_{n} d_{n} x_{n}^{\prime} \longrightarrow 0 \\
c_{n} x_{n}^{\prime \prime} \longrightarrow 0
\end{array}\right.
$$


This completes the proof of Proposition 2.7.

In terms of the normal cones $C_{N}(S)$ and $C_{M}(S)$ of [11], we get:

\section{Corollary 2.8.}

$$
\begin{aligned}
C_{N M}(S) \cap T_{N} M & =C_{N}(S) \cap T_{N} M, \\
C_{N M}(S) \cap N \times{ }_{M} T_{M} X & =C_{M}(S) \cap N \times{ }_{M} T_{M} X .
\end{aligned}
$$

The following two propositions are essential tools for the study in the next section.

Proposition 2.9. Let $V$ be a biconic open subset of $T_{N} M \times{ }_{M} T_{M} X$.

(i) Let $W$ be an open neighborhood of $V$ in $\tilde{X}_{N M}$, and set $U=\tilde{p}(W \cap \Omega)$. Then $V \cap C_{N M}(X \backslash U)=\varnothing$.

(ii) Let $U \subset X$ be an open set such that $V \cap C_{N M}(X \backslash U)=\varnothing$. Then $\tilde{p}^{-1}(U) \sqcup V$ is an open neighborhood of $V$ in $\Omega \sqcup\left(T_{N} M \times{ }_{M} T_{M} X\right)$.

Proof. The proof is almost the same as that of Proposition 4.1.3 of [11], and we shall not repeat it.

The next proposition is analogous to Proposition 4.1 .4 of [11].

Proposition 2.10. Assume (2.28) and (2.29):

$$
T_{N} M \times{ }_{M} T_{M} X \simeq \mathbb{R}_{v^{\prime}}^{n^{\prime}} \times \mathbb{R}_{v^{\prime \prime}}^{n^{\prime \prime}} \times \mathbb{R}_{x^{\prime \prime \prime}}^{n^{\prime \prime \prime}} .
$$

Let $V=V^{\prime} \times V^{\prime \prime} \times V^{\prime \prime \prime} \subset T_{N} M \times{ }_{M} T_{M} X$ be an open subset of the product-type with $V^{\prime} \varsubsetneqq \mathbb{R}_{v^{\prime}}^{n^{\prime}}$ and $V^{\prime \prime} \varsubsetneqq \mathbb{R}_{v^{\prime \prime}}^{n^{\prime \prime}}$ are conic open subsets and $V^{\prime \prime \prime} \subset \mathbb{R}_{x^{\prime \prime \prime}}^{n^{\prime \prime \prime}}$ is an open subset. Then, the family of open neighborhoods $W$ of $V$ in $\tilde{X}_{N M}$ which satisfy the following condition (2.40) forms a fundamental neighborhood system of $V$.

$$
R \Gamma\left(F_{x}, \mathbb{C}_{F_{x}}\right) \simeq \mathbb{C} \text { if } F_{x} \neq \emptyset \text { for every } x \in X,
$$

where $F_{x}:=\tilde{p}^{-1}(x) \cap(W \cap \Omega)$.

Proof. Set $\dot{\Omega}:=\Omega \cap\left\{x^{\prime} \neq 0, x^{\prime \prime} \neq 0\right\} \quad$ and $S:=\Omega \cap\left\{\left|x^{\prime}\right|=1,\left|x^{\prime \prime}\right|=1\right\}$. Then $\dot{\Omega}$ is closed under the $\left(\mathbb{R}^{+}\right)^{2}$-action $(2.25)$ on $\tilde{X}_{N M}$ and

$$
S \simeq \dot{\Omega} /\left(\mathbb{R}^{+}\right)^{2} .
$$

For a point $p_{0}=\left(x^{\prime}, x^{\prime \prime}, x^{\prime \prime \prime}, t_{1}, t_{2}\right) \in S$ which satisfies $\left|x^{\prime}\right|=\left|x^{\prime \prime}\right|=1$ and $t_{1}, t_{2}>0$, the orbit of the $\left(\mathbb{R}^{+}\right)^{2}$-action through $p_{0}$ can be expressed as follows:

$$
O_{p_{0}}:=\left\{\left(c d x^{\prime}, c x^{\prime \prime}, x^{\prime \prime \prime}, \frac{t_{1}}{c}, \frac{t_{2}}{d}\right) ;(c, d) \in\left(\mathbb{R}^{+}\right)^{2}\right\}
$$




$$
\simeq\left(\mathbb{R}^{+}\right)^{2}
$$

We identify $O_{p_{0}}$ with $\left(\mathbb{R}^{+}\right)_{(c, d)}^{2}$ and the original point $p_{0} \in S$ with the point $(c, d)=(1,1)$ of $\left(\mathbb{R}^{+}\right)^{2}$. Now let us take an arbitrary open neighborhood $W$ of $V$. One may assume $W \cap \Omega \subset \dot{\Omega}$, because $\{0\} \notin V^{\prime}$ and $\{0\} \notin V^{\prime \prime}$. For $p_{0} \in S \cap W, O_{p_{0}} \cap W$ corresponds to an open subset of $\left(\mathbb{R}^{+}\right)^{2}$ containing the point $(1,1)$, and set:

$$
\begin{aligned}
& \tilde{W}_{p_{0}}:=\left\{\text { maximal star-shaped open subset of }\left(\mathbb{R}^{+}\right)^{2}\right. \\
& \text { centered at the point } \left.(1,1) \text { in } O_{p_{0}} \cap W\right\} \text {. }
\end{aligned}
$$

Define a subset $\tilde{W}$ of $W \cap \Omega$ by:

$$
\tilde{W}:=\bigcup_{p_{0} \in S \cap W} \tilde{W}_{p_{0}} .
$$

Claim 1. $\tilde{W}$ is an open subset of $W \cap \Omega$.

Proof. Take an arbitrary point $q_{0} \in \tilde{W}_{p_{0}}\left(p_{0} \in S \cap W\right)$, and an open subset $U_{q_{0}}$ of $O_{p_{0}} \simeq\left(\mathbb{R}^{+}\right)^{2}$, such that:

$$
\left[p_{0}, q_{0}\right] \subset U_{q_{0}} \subset \subset \tilde{W}_{p_{0}} \subset O_{p_{0}},
$$

where $\left[p_{0}, q_{0}\right]$ denotes the line segment connecting the points $p_{0}$ and $q_{0}$ in $O_{p_{0}} \simeq\left(\mathbb{R}^{+}\right)^{2}$.

Now consider the continuous map:

$$
\phi: S \times\left(\mathbb{R}^{+}\right)^{2} \longrightarrow \tilde{X}_{N M}
$$

defined by

$$
\left(\left(x^{\prime}, x^{\prime \prime}, x^{\prime \prime \prime}, t_{1}, t_{2}\right),(c, d)\right) \longmapsto\left(c d x^{\prime}, c x^{\prime \prime}, x^{\prime \prime \prime},{ }^{c_{1}}, \frac{t_{2}}{d}\right)
$$

Then we have $\phi\left(\left\{p_{0}\right\} \times \bar{U}_{q_{0}}\right) \subset W$. Since $\phi\left(\left\{p_{0}\right\} \times \bar{U}_{q_{0}}\right)$ is compact in $W$, there exists an open neighborhood $V_{p_{0}}$ of $p_{0}$ in $S$ such that:

$$
\left\{\begin{array}{l}
p \in V_{p_{0}} \subset S \cap W, \text { and } \\
\phi\left(V_{p_{0}} \times \bar{U}_{q_{0}}\right) \subset W
\end{array}\right.
$$

Therefore $\phi$ induces a diffeomorphism between $V_{p_{0}} \times U_{q_{0}}$ and $\phi\left(V_{p_{0}} \times U_{q_{0}}\right)$, and $\phi\left(V_{p_{0}} \times U_{q_{0}}\right)$ is an open subset of $W$ which contains $q_{0}$. By the definition of $\tilde{W}$, we see that there exists an open neighborhood $U$ of $q_{0}$ in $\tilde{X}_{N M}$, such that $U \subset \tilde{W}$. This completes the proof of Claim 1 .

Claim 2. For every $x_{0}=\left(x_{0}^{\prime}, x_{0}^{\prime \prime}, x_{0}^{\prime \prime \prime}\right) \in V$ (hence $x_{0}^{\prime} \neq 0$ and $x_{0}^{\prime \prime} \neq 0$ ), there exists an open neighborhood $U_{x_{0}}$ of $\left(x_{0}, 0,0\right)$ in $\tilde{X}_{N M}$ such that: 


$$
U_{x_{0}} \cap \Omega \subset \tilde{W}
$$

Proof. Let us take an open neighborhood $D_{x_{0}}$ of $x_{0}$ in $V$ such that:

$$
D_{x_{0}}=D_{x_{0}^{\prime}} \times D_{x_{0}^{\prime \prime}} \times D_{x_{0}^{\prime \prime \prime}}
$$

where $D_{x_{0}^{\prime}} \subset \subset V^{\prime}, D_{x_{0}^{\prime \prime}} \subset \subset V^{\prime \prime}$ and $D_{x_{0}^{\prime \prime \prime}} \subset \subset V^{\prime \prime \prime}$ are open balls centered at $x_{0}^{\prime}, x_{0}^{\prime \prime}$ and $x_{0}^{\prime \prime \prime}$ respectively. We only have to find $\delta>0$ such that:

$$
D_{x_{0}} \times\left\{0<t_{1}<\delta\right\} \times\left\{0<t_{2}<\delta\right\} \subset \tilde{W} .
$$

First let us define $K_{S}$ as the image of $\bar{D}_{x_{0}}=\bar{D}_{x_{0}^{\prime}} \times \bar{D}_{x_{0}^{\prime \prime}} \times \bar{D}_{x_{0}^{\prime \prime \prime}}$ by the natural continuous mapping:

$$
\phi_{S}:\left(\mathbb{R}_{x^{\prime}}^{n^{\prime}} \backslash\{0\}\right) \times\left(\mathbb{R}_{x^{\prime \prime}}^{n^{\prime \prime}} \backslash\{0\}\right) \times \mathbb{R}_{x^{\prime \prime \prime}}^{n^{\prime \prime \prime}} \longrightarrow S^{n^{\prime}-1} \times S^{n^{\prime \prime}-1} \times \mathbb{R}_{x^{\prime \prime \prime}}^{n^{\prime \prime \prime}}
$$

defined by

$$
\left(x^{\prime}, x^{\prime \prime}, x^{\prime \prime \prime}\right) \longmapsto\left(\frac{x^{\prime}}{\left|x^{\prime}\right|}, \frac{x^{\prime \prime}}{\left|x^{\prime \prime}\right|}, x^{\prime \prime \prime}\right)
$$

and define $\hat{K}_{\left(\mathbb{R}^{+}\right)^{2}}$ as the image of $\bar{D}_{x_{0}^{\prime}} \times \bar{D}_{x_{0}^{\prime \prime}}$ by the continuous map:

$$
\phi_{\left(\mathbb{R}^{+}\right)^{2}}:\left(\mathbb{R}_{x^{\prime}}^{n^{\prime}} \backslash\{0\}\right) \times\left(\mathbb{R}_{x^{\prime \prime}}^{n^{\prime \prime}} \backslash\{0\}\right) \longrightarrow\left(\mathbb{R}^{+}\right)^{2}
$$

defined by

$$
\left(x^{\prime}, x^{\prime \prime}\right) \longmapsto\left(\left|x^{\prime \prime}\right|, \frac{\left|x^{\prime}\right|}{\left|x^{\prime \prime}\right|}\right) .
$$

Next take an arbitrary compact star-shaped subset $K_{\left(\mathbb{R}^{+}\right)^{2}}$ in $\left(\mathbb{R}^{+}\right)^{2}$ centered at $(1,1)$, such that:

$$
\{(1,1)\} \cup \hat{K}_{\left(\mathbb{R}^{+}\right)^{2}} \subset K_{\left(\mathbb{R}^{+}\right)^{2}},
$$

and consider the continuous map:

$$
\psi:\left(S^{n^{\prime}-1} \times S^{n^{\prime \prime}-1} \times \mathbb{R}_{x^{\prime \prime \prime}}^{n^{\prime \prime \prime}}\right) \times\left(\mathbb{R}^{+}\right)^{2} \longrightarrow \mathbb{R}_{x^{\prime}}^{n^{\prime}} \times \mathbb{R}_{x^{\prime \prime}}^{n^{\prime \prime}} \times \mathbb{R}_{x^{\prime \prime \prime}}^{n^{\prime \prime \prime}}
$$

defined by

$$
\left(\left(y^{\prime}, y^{\prime \prime}, y^{\prime \prime \prime}\right),(c, d)\right) \longmapsto\left(c d y^{\prime}, c y^{\prime \prime}, y^{\prime \prime \prime}\right) .
$$

Then $K:=\psi\left(K_{S} \times K_{\left(\mathbb{R}^{+}\right)^{2}}\right)$ is compact by definition, and it satisfies :

$$
D_{x_{0}} \subset K \subset \subset V \text {. }
$$

Since $W$ is an open neighborhood of $V$ in $\tilde{X}_{N M}$, there exists $\varepsilon_{1}>0$ such that: 


$$
K \times\left\{0<t_{1}<\varepsilon_{1}\right\} \times\left\{0<t_{2}<\varepsilon_{1}\right\} \subset W .
$$

By virtue of the compactness of $K_{\left(\mathbb{R}^{+}\right)^{2}}$ in $\left(\mathbb{R}^{+}\right)^{2}$, there exists $\varepsilon_{2}>0$ such that:

$$
\left\{\left(c d y^{\prime}, c y^{\prime \prime}, y^{\prime \prime \prime}, \frac{t_{1}}{c}, \frac{t_{2}}{d}\right) ;\left(y^{\prime}, y^{\prime \prime}, y^{\prime \prime \prime}\right) \in K_{S},(c, d) \in K_{\left(\mathbb{R}^{+}\right)^{2}}, 0<t_{1}, t_{2}<\varepsilon_{2}\right\} \subset W .
$$

Now we will complete the proof of Claim 2. For $\left(x^{\prime}, x^{\prime \prime}, x^{\prime \prime \prime}\right) \in D_{x_{0}}$, take the points :

$$
\left(\frac{x^{\prime}}{\left|x^{\prime}\right|}, \frac{x^{\prime \prime}}{\left|x^{\prime \prime}\right|}, x^{\prime \prime \prime}\right) \in K_{S} \quad \text { and } \quad\left(\left|x^{\prime \prime}\right|, \frac{\left|x^{\prime}\right|}{\left|x^{\prime \prime}\right|}\right) \in \hat{K}_{\left(\mathbb{B}^{+}\right)^{2}} \subset K_{\left(\mathbb{R}^{+}\right)^{2}}
$$

The star-shapedness of $K_{\left(\mathbb{R}^{+}\right)^{2}}$ allows us to joint the points $(1,1)$ and $\left(\left|x^{\prime \prime}\right|, \frac{\left|x^{\prime}\right|}{\left|x^{\prime \prime}\right|}\right)$ in $K_{\left(\mathbb{R}^{+}\right)^{2}}$ by a line segment, and we have:

$$
\left(x^{\prime}, x^{\prime \prime}, x^{\prime \prime \prime}, \frac{t_{1}}{\left|x^{\prime \prime}\right|}, \frac{t_{2}}{\left|x^{\prime}\right| /\left|x^{\prime \prime}\right|}\right) \in \tilde{W}
$$

for $0<t_{1}, t_{2}<\varepsilon_{2}$. Since we may assume there exists $c>0$ such that:

$$
\left|x^{\prime \prime}\right|<c \text { and } \frac{\left|x^{\prime}\right|}{\left|x^{\prime \prime}\right|}<c \text { for every } x \in D_{x_{0}},
$$

we have by setting $\delta:=\frac{\varepsilon_{2}}{c}$ :

$$
D_{x_{0}} \times\left\{0<t_{1}<\delta\right\} \times\left\{0<t_{2}<\delta\right\} \subset \tilde{W} .
$$

Thus the proof of Claim 2 is also complete. Now we will finish the proof of the proposition. By Claim 2, we find an open neighborhood $U_{x_{0}}$ of $\left(x_{0}, 0,0\right)$ in $\tilde{X}_{N M}$ for every $x_{0} \in V$, such that:

$$
U_{x_{0}} \subset W \text { and } U_{x_{0}} \cap \Omega \subset \tilde{W} \text {. }
$$

Set :

$$
\hat{W}:=\tilde{W} \cup\left(\bigcup_{x_{0} \in V} U_{x_{0}}\right)
$$

Then it is an open neighborhood of $V$ in $\tilde{X}_{N M}$ and satisfies the condition:

$$
\left\{\begin{array}{l}
\hat{W} \subset W \\
\hat{W} \cap \Omega=\tilde{W}
\end{array}\right.
$$

Therefore for every $x \in X, F_{x}:=\tilde{p}^{-1}(x) \cap(\hat{W} \cap \Omega)$ is a star-shaped open subset 
of $\left(\mathbb{R}^{+}\right)^{2}$ or an empty set.

\section{§3. Bispecialization Functor}

\subsection{Functorial properties of the functor $v_{N M}$}

In this section, we shall define the functor $v_{N M}$ of bispecialization along $(M, N)$, and investigate its functorial properties. From now on, we will frequently use the terminology of derived categories. Let $A$ be a commutative ring with finite global dimension. We denote by $\mathbf{D}^{\mathrm{b}}(X)$ the derived category of complexes of sheaves of $A_{X}$-module with bounded cohomologies on a manifold $X$.

Definition 3.1. Let $F \in \mathbf{D}^{\mathrm{b}}(X)$. One sets :

$$
v_{N M}(F):=s^{-1} R j_{*} \tilde{p}^{-1} F,
$$

and says that $v_{N M}(F)$ is the bispecialization of $F$ along $(M, N)$.

As we see in the next proposition, the functor $v_{N M}$ is a generalization of the usual one $v_{M}$.

Proposition 3.2. Let $M=N$ and identify $T_{M} M \times{ }_{M} T_{M} X$ with $T_{M} X$. Then for every $F \in \mathbf{D}^{\mathrm{b}}(X)$, we have a canonical isomorphism:

$$
v_{M}(F) \stackrel{\sim}{\longrightarrow} v_{M M}(F)
$$

Proof. First consider the natural morphism $q: \tilde{X}_{M M} \rightarrow \tilde{X}_{M}$ given by $\left(t_{1}, t_{2}\right) \mapsto t_{1} t_{2}$ and the commutative diagram:

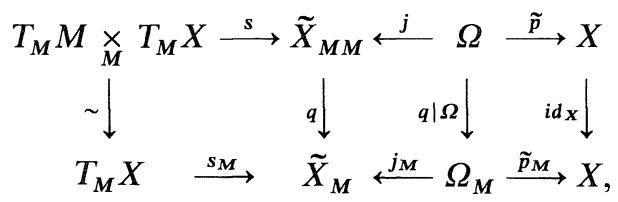

where $\Omega_{M}$ is the open subset of $\tilde{X}_{M}$ defined by $\Omega_{M}:=\{t>0\}$. Then we can construct the morphism:

$$
v_{M}(F) \longrightarrow v_{M M}(F)
$$

by the same way as in the proof of Proposition 4.2.5 of [11]. The stalks of $v_{M M}(F)$ coincide with those of $v_{M}(F)$ because we can easily obtain the results corresponding to Proposition 4.1.3 and Proposition 4.1.4 of [11] for the case $\tilde{X}_{M M} \rightarrow X$. This completes the proof. 
Let $(X, M, N)$ and $\left(X^{\prime}, M^{\prime}, N^{\prime}\right)$ be triplets of manifolds, and $f$ be a morphism from $X^{\prime}$ to $X$ such that

$$
\left\{\begin{array}{l}
f\left(M^{\prime}\right) \subset M \\
f\left(N^{\prime}\right) \subset N .
\end{array}\right.
$$

For the sake of simplicity, we shall often write:

$$
f:\left(X^{\prime}, M^{\prime}, N^{\prime}\right) \longrightarrow(X, M, N) .
$$

If we take a local coordinate system $\left(x^{\prime}, x^{\prime \prime}, x^{\prime \prime \prime}\right)\left(\right.$ resp. $\left.\left(y^{\prime}, y^{\prime \prime}, y^{\prime \prime \prime}\right)\right)$ of $(X, M, N)$ (resp. $\left(X^{\prime}, M^{\prime}, N^{\prime}\right)$ ) and set

$$
f\left(y^{\prime}, y^{\prime \prime}, y^{\prime \prime \prime}\right)=\left(\phi^{\prime}(y), \phi^{\prime \prime}(y), \phi^{\prime \prime \prime}(y)\right),
$$

then we can define a morphism $\tilde{f}^{\prime}$ from $\tilde{X}_{N^{\prime} M^{\prime}}^{\prime}$ to $\tilde{X}_{N M}$ as follows :

$$
\left\{\begin{aligned}
x^{\prime} & =\frac{1}{t_{1} t_{2}} \phi^{\prime}\left(t_{1} t_{2} y^{\prime}, t_{1} y^{\prime \prime}, y^{\prime \prime \prime}\right) \\
x^{\prime \prime} & =\frac{1}{t_{1}} \phi^{\prime \prime}\left(t_{1} t_{2} y^{\prime}, t_{1} y^{\prime \prime}, y^{\prime \prime \prime}\right) \\
x^{\prime \prime \prime} & =\phi^{\prime \prime \prime}\left(t_{1} t_{2} y^{\prime}, t_{1} y^{\prime \prime}, y^{\prime \prime \prime}\right) \\
t_{1} & =t_{1} \\
t_{2} & =t_{2}
\end{aligned}\right.
$$

Restricting it to $\left\{t_{1}=t_{2}=0\right\}=T_{N^{\prime}} M^{\prime} \underset{M^{\prime}}{\times} T_{M^{\prime}} X^{\prime}$, we get a morphism $T f$ :

$$
\left\{\begin{aligned}
v^{\prime} & =\frac{\partial \phi^{\prime}}{\partial x^{\prime}}\left(0,0, y^{\prime \prime \prime}\right) \cdot u^{\prime} \\
v^{\prime \prime} & =\frac{\partial \phi^{\prime \prime}}{\partial x^{\prime \prime}}\left(0,0, y^{\prime \prime \prime}\right) \cdot u^{\prime \prime} \\
x^{\prime \prime \prime} & =\phi^{\prime \prime \prime}\left(0,0, y^{\prime \prime \prime}\right)
\end{aligned}\right.
$$

where $\left(v^{\prime} \frac{\partial}{\partial x^{\prime}}, v^{\prime \prime} \frac{\partial}{\partial x^{\prime \prime}}, x^{\prime \prime \prime}\right)\left(\operatorname{resp} .\left(u^{\prime} \frac{\partial}{\partial y^{\prime}}, u^{\prime \prime} \frac{\partial}{\partial y^{\prime \prime}}, y^{\prime \prime \prime}\right)\right)$ is the associated coordinate system of $T_{N} M \underset{M}{\times} T_{M} X$ (resp. $T_{N^{\prime}} M^{\prime} \underset{M^{\prime}}{\times} T_{M^{\prime}} X^{\prime}$ ). Thus, we get a commutative diagram below: 


$$
\begin{aligned}
& T_{N^{\prime}} M^{\prime} \underset{M^{\prime}}{\times} T_{M^{\prime}} X^{\prime} \stackrel{s_{X^{\prime}}}{\longrightarrow} \tilde{X}_{N^{\prime} M^{\prime}}^{\prime} \stackrel{j_{X^{\prime}}}{\longleftarrow} \Omega_{X^{\prime}} \stackrel{\tilde{p}_{X^{\prime}}}{\longrightarrow} X^{\prime} \\
& T_{N} M \underset{M}{\times} T_{M} X \stackrel{s_{X}}{\longrightarrow} \tilde{X}_{N M} \stackrel{j_{X}}{\longleftarrow} \Omega_{X} \stackrel{\tilde{p}_{X}}{\longrightarrow} X,
\end{aligned}
$$

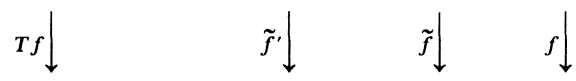

in which all squares are Cartesian.

By paraphrasing the arguments of [11], one obtains functorial properties of the functor $v_{N M}$.

Proposition 3.3. Let $G \in \mathbf{D}^{\mathrm{b}}\left(X^{\prime}\right)$.

(i) There exists a commutative diagram:

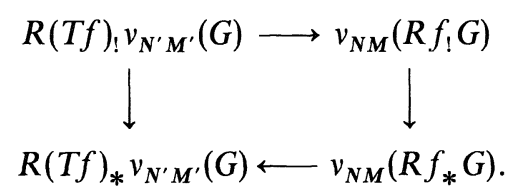

(ii) Assume $f: X^{\prime} \rightarrow X$ is a closed embedding, $f^{-1}(M)=M^{\prime}, f^{-1}(N)=N^{\prime}$, and $f: X^{\prime} \rightarrow X$ and $\left.f\right|_{M^{\prime}}: M^{\prime} \rightarrow M$ are clean w.r.t. $M$ and $N$ respectively. Then all these morphisms are isomorphisms.

Proposition 3.4. Let $F \in \mathrm{D}^{\mathrm{b}}(X)$.

(i) There are canonical morphisms:

$$
\left\{\begin{array}{l}
\alpha:(T f)^{-1} v_{N M}(F) \longrightarrow v_{N^{\prime} M^{\prime}}\left(f^{-1} F\right), \\
\beta: v_{N^{\prime} M^{\prime}}\left(f^{!} F\right) \longrightarrow(T f)^{!} v_{N M}(F),
\end{array}\right.
$$

such that the following diagram commutes:

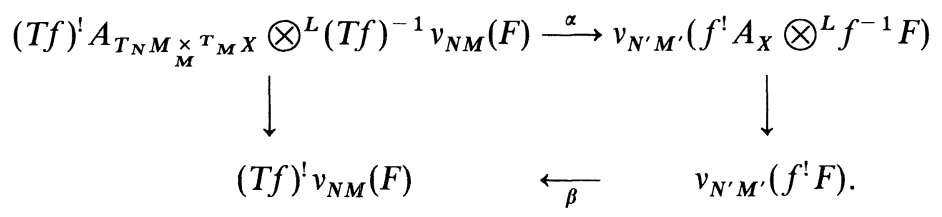

(ii) If $f: X^{\prime} \rightarrow X,\left.f\right|_{M^{\prime}}: M^{\prime} \rightarrow M$ and $\left.f\right|_{N^{\prime}}: N^{\prime} \rightarrow N$ are all smooth, then all these morphisms are isomorphisms.

Proposition 3.5. Let $F \in \mathbf{D}^{\mathrm{b}}(X)$ and $G \in \mathbf{D}^{\mathrm{b}}\left(X^{\prime}\right)$. Then we have a canonical morphism:

$$
v_{N M}(F) \bigotimes^{L} v_{N^{\prime} M^{\prime}}(G) \longrightarrow v_{\left(N \times N^{\prime}\right)\left(M \times M^{\prime}\right)}\left(F \bigotimes^{L} G\right)
$$

in $\mathbf{D}^{\mathrm{b}}\left(T_{\left(N \times N^{\prime}\right)}\left(M \times M^{\prime}\right) \underset{\left(M \times M^{\prime}\right)}{\times} T_{\left(M \times M^{\prime}\right)}\left(X \times X^{\prime}\right)\right)$. 
Proposition 3.6. Let $F, G \in \mathbf{D}^{\mathrm{b}}(X)$. Then we have a canonical morphism:

$$
v_{N M}(F) \otimes \otimes^{L} v_{N M}(G) \longrightarrow v_{N M}\left(F \otimes^{L} G\right) \text {. }
$$

As. a particular case, consider the morphism $f=\mathrm{id}_{X}:(X, M, N) \rightarrow(X, M, M)$ and the commutative diagram:

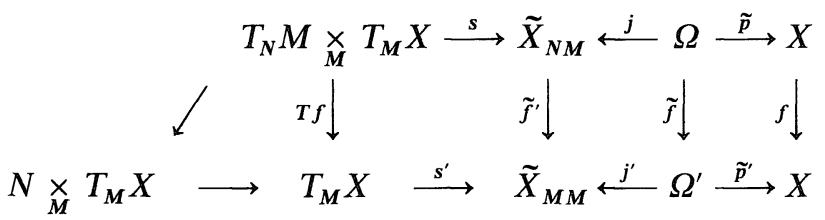

in which all squares are Cartesian and $\Omega^{\prime}$ is the open subset of $\tilde{X}_{M M}$ defined by $\Omega^{\prime}:=\left\{t_{1}>0, t_{2}>0\right\}$. Then by Proposition 3.4 , we get a natural morphism:

$$
\alpha_{0}:(T f)^{-1} v_{M M}(F) \longrightarrow v_{N M}(F)
$$

Next, consider the following commutative diagram:

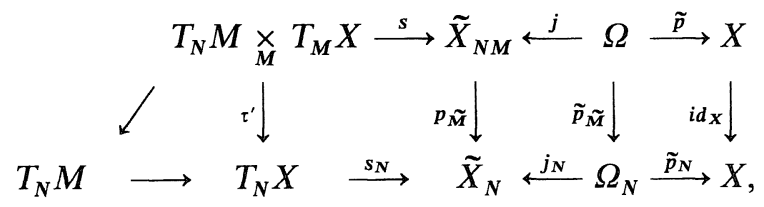

where $\Omega_{N}$ is the open subset of $\tilde{X}_{N}$ defined by $\Omega_{N}:=\left\{t_{1}>0\right\}$. Then we can construct a canonical morphism:

$$
\beta_{0}: \tau^{\prime-1} v_{N}(F) \longrightarrow v_{N M}(F)
$$

by the same way as in the proof of Proposition 4.2.5 of [11].

Theorem 3.7. The morphisms $\alpha:=\left.\alpha_{0}\right|_{N{ }_{M} T_{M} X}$ and $\beta:=\left.\beta_{0}\right|_{T_{N} M}$ are isomorphisms. In view of Proposition 3.2, for $F \in \mathbf{D}^{\mathrm{b}}(X)$ we have natural isomorphisms:

$$
\begin{gathered}
\alpha:\left.\left.v_{M}(F)\right|_{N_{M} \times T_{M} X} \stackrel{\sim}{\longrightarrow} v_{N M}(F)\right|_{N_{M} \times T_{M} X}, \\
\beta:\left.\left.v_{N}(F)\right|_{T_{N} M} \stackrel{\sim}{\longrightarrow} v_{N M}(F)\right|_{T_{N} M} .
\end{gathered}
$$

Proof. First let us show that $\alpha$ is an isomorphism, i.e.

$$
\alpha:\left.\left.\tilde{f}^{\prime-1} R j^{\prime} \tilde{p}^{\prime-1} F\right|_{N_{M} \times T_{M} X} \stackrel{\sim}{\longrightarrow} R j_{*} \tilde{f}^{-1} \tilde{p}^{\prime-1} F\right|_{N_{M} \times T_{M} X} .
$$

For $p_{0}=\left(x_{0}^{\prime}, 0,0,0,0\right) \in N \underset{M}{\times} T_{M} X \subset \tilde{X}_{N M}$ and for every $j \in \mathbb{Z}$ : 


$$
\begin{aligned}
H^{j}\left[R j_{*} \tilde{f}^{-1} \tilde{p}^{\prime-1} F\right]_{p_{0}} & \simeq \varliminf_{\varepsilon>0} H^{j}\left(U_{\varepsilon} ; \tilde{f}^{-1} \tilde{p}^{\prime-1} F\right) \\
& \simeq \varliminf_{\varepsilon>0} H^{j}\left(\tilde{f}\left(U_{\varepsilon}\right) ; \tilde{p}^{\prime-1} F\right),
\end{aligned}
$$

where

$$
\begin{aligned}
U_{\varepsilon}:= & \left\{x^{\prime} ;\left|x^{\prime}-x_{0}^{\prime}\right|<\varepsilon\right\} \times\left\{\left|x^{\prime \prime}\right|<\varepsilon\right\} \times\left\{\left|x^{\prime \prime \prime}\right|<\varepsilon\right\} \\
& \times\left\{0<t_{1}<\varepsilon, 0<t_{2}<\varepsilon\right\}
\end{aligned}
$$

and we have used the fact that $\tilde{f}$ is a diffeomorphism between $\Omega$ and $\Omega^{\prime}$ in the second equality. Since $\tilde{f}$ is explicitly described by:

$$
\left(x^{\prime}, x^{\prime \prime}, x^{\prime \prime \prime}, t_{1}, t_{2}\right) \longmapsto\left(x^{\prime}, t_{1} x^{\prime \prime}, x^{\prime \prime \prime}, t_{1}, t_{2}\right)
$$

by using a local coordinate system,

$$
\begin{gathered}
\tilde{f}\left(U_{\varepsilon}\right)=\left\{\left(x^{\prime}, x^{\prime \prime}, x^{\prime \prime \prime}, t_{1}, t_{2}\right) \in \tilde{X}_{M M} ;\left|x^{\prime}-x_{0}^{\prime}\right|<\varepsilon,\left|x^{\prime \prime}\right|<\varepsilon t_{1},\right. \\
\left.\left|x^{\prime \prime \prime}\right|<\varepsilon, 0<t_{1}<\varepsilon, 0<t_{2}<\varepsilon\right\} \subset \Omega^{\prime} .
\end{gathered}
$$

Now set

$$
\begin{aligned}
V_{\varepsilon}:= & \left\{\left(x^{\prime}, x^{\prime \prime}, x^{\prime \prime \prime}, t_{1}, t_{2}\right) \in \tilde{X}_{M M} ;\left|x^{\prime}-x_{0}^{\prime}\right|<\varepsilon,\left|x^{\prime \prime}\right|<\varepsilon^{2},\right. \\
& \left.\left|x^{\prime \prime \prime}\right|<\varepsilon, 0<t_{1}<\varepsilon, 0<t_{2}<\varepsilon\right\} \subset \Omega^{\prime} .
\end{aligned}
$$

Then $V_{\varepsilon} \supset \tilde{f}\left(U_{\varepsilon}\right)$. On the other hand, we have:

$$
\left.H^{j} \mid \tilde{f}^{\prime-1} R j^{\prime}{ }_{*} \tilde{p}^{\prime-1} F\right]_{p_{0}} \simeq H^{j}\left[R j^{\prime}{ }_{*} \tilde{p}^{\prime-1} F\right]_{\tilde{f}^{\prime}\left(p_{0}\right)} \simeq \varliminf_{\varepsilon>0} H^{j}\left(V_{\varepsilon} ; \tilde{p}^{\prime-1} F\right)
$$

for every $j \in \mathbb{Z}$, because $\tilde{f}^{\prime}\left(p_{0}\right)=\left(x_{0}^{\prime}, 0,0,0,0\right)$ in $\tilde{X}_{M M}$. Thus it remains to show the isomorphism:

$$
R \Gamma\left(V_{\varepsilon} ; \tilde{p}^{\prime-1} F\right) \stackrel{\sim}{\longrightarrow} R \Gamma\left(\tilde{f}\left(U_{\varepsilon}\right) ; \tilde{p}^{\prime-1} F\right)
$$

for every $\varepsilon>0$. For $x_{1}=\left(x_{1}^{\prime}, x_{1}^{\prime \prime}, x_{1}^{\prime \prime \prime}\right) \in X$, the inverse image $\tilde{p}^{\prime-1}\left(x_{1}\right)$ in $\Omega^{\prime} \subset \tilde{X}_{M M}$ is expressed in the following way:

$$
\tilde{p}^{\prime-1}\left(x_{1}\right)=\left\{\left(\frac{x_{1}^{\prime}}{t_{1} t_{2}}, x_{1}^{\prime \prime}, x_{1}^{\prime \prime \prime}, t_{1}, t_{2}\right) ;\left(t_{1}, t_{2}\right) \in\left(\mathbb{R}^{+}\right)^{2}\right\} \text {. }
$$

We can find that $\tilde{p}^{\prime-1}\left(x_{1}\right) \cap V_{\varepsilon}$ and $\tilde{p}^{\prime-1}\left(x_{1}\right) \cap \tilde{f}\left(U_{\varepsilon}\right)$ are isomorphic to open rectangles in $\left(\mathbb{R}^{+}\right)^{2}$ if they are not empty. Moreover we have:

$$
\tilde{p}^{\prime-1}\left(x_{1}\right) \cap V_{\varepsilon} \neq \emptyset \Longleftrightarrow \tilde{p}^{\prime-1}\left(x_{1}\right) \cap \tilde{f}\left(U_{\varepsilon}\right) \neq \varnothing .
$$

Therefore, the required isomorphism is deduced from a slight modification of Corollary 3.7.3 of [11], and the morphism $\alpha$ is an isomorphism. 
Next, we shall show that $\beta$ is an isomorphism, i.e.

$$
\beta:\left.\left.p_{\tilde{M}}^{-1} R j_{N *} \tilde{p}_{N}^{-1} F\right|_{T_{N} M} \stackrel{\sim}{\longrightarrow} R j_{*} \tilde{p}_{\tilde{M}}^{-1} \tilde{p}_{N}^{-1} F\right|_{T_{N} M} .
$$

For $q_{0}=\left(0, x_{0}^{\prime \prime}, 0,0,0\right) \in T_{N} M \subset \tilde{X}_{N M}$ and for every $j \in \mathbb{Z}$ :

$$
H^{j}\left[R j_{*} \tilde{p}_{\tilde{M}}^{-1} \tilde{p}_{N}^{-1} F\right]_{q_{0}} \simeq \varliminf_{\varepsilon>0} H^{j}\left(W_{\varepsilon} ; \tilde{p}_{\tilde{M}}^{-1} \tilde{p}_{N}^{-1} F\right)
$$

where

$$
\begin{aligned}
W_{\varepsilon}:= & \left\{\left|x^{\prime}\right|<\varepsilon\right\} \times\left\{x^{\prime \prime} ;\left|x^{\prime \prime}-x_{0}^{\prime \prime}\right|<\varepsilon\right\} \times\left\{\left|x^{\prime \prime \prime}\right|<\varepsilon\right\} \\
& \times\left\{0<t_{1}<\varepsilon, 0<t_{2}<\varepsilon\right\} \subset \Omega \subset \tilde{X}_{N M} .
\end{aligned}
$$

Now we have:

$$
\begin{aligned}
\tilde{p}_{\tilde{M}}\left(W_{\varepsilon}\right)= & \left\{\left|x^{\prime}\right|<\varepsilon^{2}\right\} \times\left\{x^{\prime \prime} ;\left|x^{\prime \prime}-x_{0}^{\prime \prime}\right|<\varepsilon\right\} \times\left\{\left|x^{\prime \prime \prime}\right|<\varepsilon\right\} \\
& \times\left\{0<t_{1}<\varepsilon\right\} \subset \Omega_{N} \subset \tilde{X}_{N},
\end{aligned}
$$

because $\tilde{p}_{\tilde{M}}: \Omega \rightarrow \Omega_{N}$ is explicitly described by:

$$
\left(x^{\prime}, x^{\prime \prime}, x^{\prime \prime \prime}, t_{1}, t_{2}\right) \longmapsto\left(t_{2} x^{\prime}, x^{\prime \prime}, x^{\prime \prime \prime}, t_{1}\right)
$$

in a local coordinate system. Since $\left.\tilde{p}_{\tilde{M}}\right|_{W_{\varepsilon}}=\left.p_{\tilde{M}}\right|_{W_{\varepsilon}}: W_{\varepsilon} \rightarrow p_{\tilde{M}}\left(W_{\varepsilon}\right)$ is a topological submersion with contractible fibers, we have an isomorphism:

$$
\mathrm{id} \stackrel{\sim}{\longrightarrow} R\left(\left.p_{\tilde{M}}\right|_{W_{\varepsilon}}\right)_{*}\left(\left.p_{\tilde{M}}\right|_{W_{\varepsilon}}\right)^{-1}
$$

in $\mathbf{D}^{\mathrm{b}}\left(p_{\tilde{\boldsymbol{M}}}\left(W_{\varepsilon}\right)\right)$ by Proposition 3.3.9 of [11]. Therefore, for every $j \in \mathbb{Z}$ :

$$
H^{j}\left(W_{\varepsilon} ; \tilde{p}_{\tilde{M}}^{-1} \tilde{p}_{N}^{-1} F\right) \stackrel{\sim}{\longleftarrow} H^{j}\left(p_{\tilde{M}}\left(W_{\varepsilon}\right) ; \tilde{p}_{N}^{-1} F\right) .
$$

On the other hand,

$$
\begin{aligned}
H^{j}\left[p_{\tilde{M}}^{-1} R j_{N *} \tilde{p}_{N}^{-1} F\right]_{q_{0}} & \simeq H^{j}\left(R j_{N *} \tilde{p}_{N}^{-1} F\right]_{p_{\tilde{M}}\left(q_{0}\right)} \\
& \simeq \varliminf_{\varepsilon>0} H^{j}\left(p_{\tilde{M}}\left(W_{\varepsilon}\right) ; \tilde{p}_{N}^{-1} F\right) .
\end{aligned}
$$

because $p_{\tilde{M}}\left(q_{0}\right)=\left(0, x_{0}^{\prime \prime}, 0,0\right)$ in $\tilde{X}_{N}$. This completes the proof.

\subsection{Structure of the functor $v_{N M}$}

In this section, we shall describe the global sections of $v_{N M}(F)$ on product type biconic open subsets of $T_{N} M \underset{M}{\times} T_{M} X$.

Assume: 


$$
\left\{\begin{array}{l}
X=X_{x^{\prime}}^{\prime} \times X_{x^{\prime \prime}}^{\prime \prime} \times X_{x^{\prime \prime \prime}}^{\prime \prime \prime} \\
M=\{0\} \times X_{x^{\prime \prime}}^{\prime \prime} \times X_{x^{\prime \prime \prime}}^{\prime \prime \prime}, \\
N=\{0\} \times\{0\} \times X_{x^{\prime \prime \prime}}^{\prime \prime \prime}
\end{array}\right.
$$

where $X^{\prime}, X^{\prime \prime}$ and $X^{\prime \prime \prime}$ are real finite dimensional vector spaces. Then we have:

$$
T_{N} M \times{ }_{M} T_{M} X=X_{v^{\prime}}^{\prime} \times X_{v^{\prime \prime}}^{\prime \prime} \times X_{x^{\prime \prime \prime}}^{\prime \prime \prime}
$$

Theorem 3.8. Let $F \in \mathbf{D}^{\mathrm{b}}(X)$. Then:

(i) $v_{N M}(F) \in \mathbf{D}_{\mathbb{R}^{+} \times \mathbb{R}^{+}}^{\mathrm{b}}\left(T_{N} M \times{ }_{M} T_{M} X\right)$, that is, it is a biconic object on $T_{N} M \times{ }_{M} T_{M} X$, and

$$
\operatorname{supp} v_{N M}(F) \subset C_{N M}(\operatorname{supp} F) \text {. }
$$

(ii) Let $V \subset T_{N} M \times{ }_{M} T_{M} X$ be a biconic open subset of the product type:

$$
V=V^{\prime} \times V^{\prime \prime} \times V^{\prime \prime \prime},
$$

that is, $V^{\prime} \subset X_{v^{\prime}}^{\prime}$ and $V^{\prime \prime} \subset X_{v^{\prime \prime}}^{\prime \prime}$ are conic open subsets and $V^{\prime \prime \prime} \subset X_{x^{\prime \prime \prime}}^{\prime \prime \prime}$ is an open set. Then for every $j \in \mathbb{Z}$ :

$$
H^{j}\left(V ; v_{N M}(F)\right) \simeq \underbrace{}_{C_{N M}(X \backslash U) \cap V \neq \emptyset} H^{j}(U ; F) .
$$

Proof.

(i) Obvious. (ii) Case 1. Assume $V^{\prime} \varsubsetneqq X_{v^{\prime}}^{\prime}$ and $V^{\prime \prime} \varsubsetneqq X_{v^{\prime \prime}}^{\prime \prime}$. Then the proof is almost similar to that of Theorem 4.2.3 of [11]. (Use Proposition 2.9 and Proposition 2.10. See Remark 3.3.10 of [11].)

Case 2. Assume $V^{\prime}=X_{v^{\prime}}^{\prime}$, i.e.

$$
V=X_{v^{\prime}}^{\prime} \times V^{\prime \prime} \times V^{\prime \prime \prime}
$$

Since $C_{N M}(S)$ is a biconic closed subset of $T_{N} M \times{ }_{M} T_{M} X$ for every $S \subset X$, we have:

$$
\begin{aligned}
& C_{N M}(X \backslash U) \cap V=\varnothing \\
& \Longleftrightarrow C_{N M}(X \backslash U) \cap\left(\{0\} \times V^{\prime \prime} \times V^{\prime \prime \prime}\right)=\varnothing \\
& \Longleftrightarrow C_{N}(X \backslash U) \cap\left(\{0\} \times V^{\prime \prime} \times V^{\prime \prime \prime}\right)=\varnothing
\end{aligned}
$$

for every open subset $U \subset X$, where we identified $\{0\} \times V^{\prime \prime} \times V^{\prime \prime \prime} \subset T_{N} M$ with a subset of $T_{N} X$ and we have used Corollary 2.38. For such $U \subset X$, we can construct a morphism

$$
\mathbf{A}: R \Gamma(U ; F) \longrightarrow R \Gamma\left(V ; v_{N M}(F)\right)
$$

by Proposition 2.9 (ii). (See the formula (4.2.2) of [11]). Since $v_{N M}(F)$ is a biconic object of $\mathbf{D}^{\mathrm{b}}\left(T_{N} M \times{ }_{M} T_{M} X\right)$, 


$$
R \Gamma\left(V ; v_{N M}(F)\right) \stackrel{\sim}{\longrightarrow} R \Gamma\left(\{0\} \times V^{\prime \prime} \times V^{\prime \prime \prime} ;\left.v_{N M}(F)\right|_{T_{N} M}\right),
$$

and there is a commutative diagram:

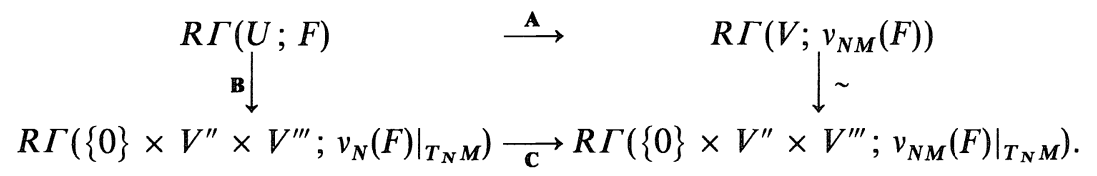

The morphism $\mathbf{C}$ is an isomorphism by Theorem 3.7, and it follows from Corollary 3.7 .3 of [11] that:

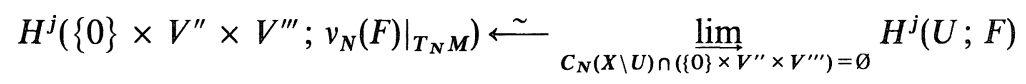

for every $j \in \mathbb{Z}$. Thus, by virtue of (3.27) and the commutativity of the diagram (3.30), we get:

$$
H^{j}\left(V ; v_{N M}(F)\right) \stackrel{\sim}{\longleftarrow} \lim _{C_{N M}(X \backslash U) \cap V=\emptyset} H^{j}(U ; F)
$$

for every $j \in \mathbb{Z}$.

Case 3. Assume $V^{\prime \prime}=X_{v^{\prime \prime}}^{\prime \prime}$, i.e.

$$
V=V^{\prime} \times X_{v^{\prime \prime}}^{\prime \prime} \times V^{\prime \prime \prime} .
$$

If we use (3.19):

$$
\left.\left.v_{N M}(F)\right|_{N_{M}^{\times} T_{M} X} \stackrel{\sim}{\longleftarrow} v_{M}(F)\right|_{N_{M} \times T_{M} X},
$$

Corollary 2.38 and Proposition 2.9 (ii), the proof proceeds similarly to that of Case 2.

Now, we consider $T_{N} M$ and $N \times{ }_{M} T_{M} X$ as zero-sections of $T_{N} M \times{ }_{M} T_{M} X$. We want to know the structure of $R \Gamma_{\left(T_{N} M\right)}\left(v_{N M}(F)\right)$ and $R \Gamma_{\left({ }_{N} \times_{M} T_{M} X\right)}\left(v_{N M}(F)\right)$. Consider the diagram (3.17). Then we have canonical morphisms:

$$
\begin{aligned}
\tau^{\prime-1} v_{N}\left(R \Gamma_{M}(F)\right) & \longrightarrow v_{N M}\left(R \Gamma_{M}(F)\right) \\
& \stackrel{\sim}{\longleftarrow} \Gamma_{\left(T_{N} M\right)}\left(v_{N M}\left(R \Gamma_{M}(F)\right)\right) \\
& \longrightarrow R \Gamma_{\left(T_{N} M\right)}\left(v_{N M}(F)\right),
\end{aligned}
$$

where the first arrow is obtained by (3.18) and the second isomorphism is a consequence of:

$$
\operatorname{supp} v_{N M}\left(R \Gamma_{M}(F)\right) \subset T_{N} M
$$


Theorem 3.9. Let $F \in \mathbf{D}^{\mathrm{b}}(X)$. Then the restriction of the morphism (3.34) to $T_{N} M \subset T_{N} M \times{ }_{M} T_{M} X$ :

$$
\left.\left.v_{N}\left(R \Gamma_{M}(F)\right)\right|_{T_{N} M} \longrightarrow R \Gamma_{\left(T_{N} M\right)}\left(v_{N M}(F)\right)\right|_{T_{N} M}
$$

is an isomorphism.

Proof. Notice:

$$
T_{N} M \underset{M}{\times} T_{M} X \backslash T_{N} M \simeq T_{N} M \underset{M}{\times} \dot{T}_{M} X,
$$

and denote this set by $A$. Then we have a sequence of morphisms:

$$
\begin{aligned}
\tau^{\prime-1} v_{N}\left(R \Gamma_{(X \backslash M)}(F)\right) & \longrightarrow v_{N M}\left(R \Gamma_{(X \backslash M)}(F)\right) \\
& \longrightarrow R \Gamma_{A}\left(v_{N M}\left(R \Gamma_{(X \backslash M)}(F)\right)\right) \\
& \stackrel{\sim}{\longleftarrow} R \Gamma_{A}\left(v_{N M}(F)\right),
\end{aligned}
$$

where the last isomorphism follows from the formula $R \Gamma_{A}\left(v_{N M}\left(R \Gamma_{M}(F)\right)\right) \simeq 0$ (cf. (3.35)). Now we have a morphism of distinguished triangles

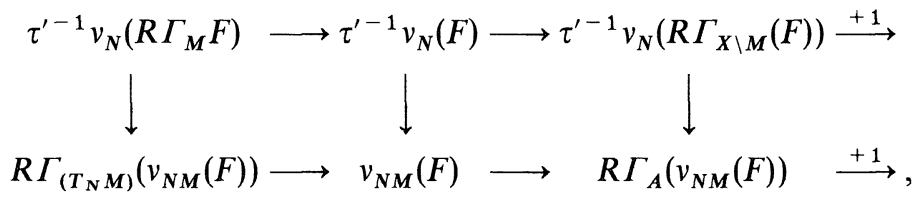

and we know by Theorem 3.7 :

$$
\left.\left.v_{N}(F)\right|_{T_{N} M} \stackrel{\sim}{\longrightarrow} v_{N M}(F)\right|_{T_{N} M} .
$$

Hence, it is enough to show

$$
\left.\left.v_{N}\left(R \Gamma_{(X \backslash M)}(F)\right)\right|_{T_{N} M} \stackrel{\sim}{\longrightarrow} R \Gamma_{A}\left(v_{N M}(F)\right)\right|_{T_{N} M} .
$$

For this purpose, suppose the condition (3.21) and (3.22), and choose:

$$
p_{0}=\left(0, x_{0}^{\prime \prime}, 0\right) \in T_{N} M \subset T_{N} M \underset{M}{\times} T_{M} X .
$$

Since $v_{N M}(F)$ is conic w.r.t. the $v^{\prime}$-variables,

$$
H^{j}\left[R \Gamma_{\left(T_{N} M \times_{M} T_{M} X \backslash T_{N} M\right)}\left(v_{N M}(F)\right)\right]_{p_{0}} \simeq \varliminf_{\varepsilon>0} H^{j}\left(U_{\varepsilon} ; \tilde{p}^{-1} F\right),
$$

where

$$
\begin{aligned}
U_{\varepsilon}:= & \left\{1-\varepsilon<\left|x^{\prime}\right|<1+\varepsilon\right\} \times\left\{\left|x^{\prime \prime}-x_{0}^{\prime \prime}\right|<\varepsilon\right\} \times\left\{\left|x^{\prime \prime \prime}\right|<\varepsilon\right\} \\
& \times\left\{0<t_{1}<\varepsilon, 0<t_{2}<\varepsilon\right\} \subset \Omega \subset \tilde{X}_{N M} .
\end{aligned}
$$


Now recall $p_{\tilde{M}}: \tilde{X}_{N M} \rightarrow \tilde{X}_{N}$ is described by

$$
\left(x^{\prime}, x^{\prime \prime}, x^{\prime \prime \prime}, t_{1}, t_{2}\right) \longmapsto\left(t_{2} x^{\prime}, x^{\prime \prime}, x^{\prime \prime \prime}, t_{1}\right),
$$

and $\left.p_{\tilde{M}}\right|_{U_{\varepsilon}}=\left.\tilde{p}_{\tilde{M}}\right|_{U_{\varepsilon}}: U_{\varepsilon} \rightarrow p_{\tilde{M}}\left(U_{\varepsilon}\right)$ is a smooth morphism with contractible fibers. Thus by Proposition 3.3.9 of [11], we have:

$$
\begin{aligned}
R \Gamma\left(p_{\tilde{M}}\left(U_{\varepsilon}\right) ; \tilde{p}_{N}^{-1} F\right) & \stackrel{\sim}{\longrightarrow} R \Gamma\left(p_{\tilde{M}}\left(U_{\varepsilon}\right) ; R\left(\left.p_{\tilde{M}}\right|_{U_{\varepsilon}}\right)_{*}\left(\left.p_{\tilde{M}}\right|_{U_{\varepsilon}}\right)^{-1} \tilde{p}_{N}^{-1} F\right) \\
& \simeq R \Gamma\left(U_{\varepsilon} ; \tilde{p}^{-1} F\right) .
\end{aligned}
$$

On the other hand,

$$
\begin{aligned}
p_{\tilde{M}}\left(U_{\varepsilon}\right)= & \left\{0<\left|x^{\prime}\right|<\varepsilon(1+\varepsilon)\right\} \times\left\{\left|x^{\prime \prime}-x_{0}^{\prime \prime}\right|<\varepsilon\right\} \\
& \times\left\{\left|x^{\prime \prime \prime}\right|<\varepsilon\right\} \times\left\{0<t_{1}<\varepsilon\right\} \subset \Omega_{N} \subset \tilde{X}_{N},
\end{aligned}
$$

and it follows from $\tilde{p}_{N}^{-1} R \Gamma_{(X \backslash M)}(F) \simeq R \Gamma_{\left(\Omega_{N} \backslash \tilde{M}\right)}\left(\tilde{p}_{N}^{-1} F\right)$ that:

$$
H^{j}\left[v_{N}\left(R \Gamma_{(X \backslash M)}(F)\right)\right]_{p_{0}} \simeq \varliminf_{\varepsilon>0} H^{j}\left(p_{\tilde{M}}\left(U_{\varepsilon}\right) ; \tilde{p}_{N}^{-1} F\right) .
$$

This completes the proof of Theorem 3.9.

Let :

$$
\imath: N \underset{M}{\times} T_{M} X \smile T_{N} M \underset{M}{\times} T_{M} X
$$

be the zero-section embedding and

$$
\rho: T_{N} X \longrightarrow N \underset{M}{\times} T_{M} X
$$

be the canonical projection. Then:

Theorem 3.10. There exists a canonical isomorphism for $F \in \mathbb{D}^{\mathrm{b}}(X)$ :

$$
R \rho_{!} v_{N}(F) \stackrel{\sim}{\longrightarrow} l^{!} v_{N M}(F)
$$

Proof. Consider the following commutative diagram:

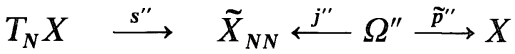

$$
\begin{aligned}
& \rho \quad T g \downarrow \quad \tilde{g}^{\prime} \downarrow \quad \tilde{g} \downarrow \quad \downarrow g=i d_{X} \\
& N \underset{M}{\times} T_{M} X \stackrel{\imath}{\longrightarrow} T_{N} M \underset{M}{\times} T_{M} X \stackrel{s}{\longrightarrow} \tilde{X}_{N M} \stackrel{j}{\longleftarrow} \Omega \stackrel{\widetilde{p}}{\longrightarrow} X .
\end{aligned}
$$

This is a particular case of the diagram (3.9) for the morphism

$$
g=\mathrm{id}_{X}:(X, N, N) \longrightarrow(X, M, N)
$$


(Note that $\tilde{g}: \Omega^{\prime \prime} \simeq \Omega$ is an isomorphism). By virtue of Proposition 3.3 (i), we have a canonical morphism:

$$
R(T g) v_{N N}(F) \longrightarrow v_{N M}(F) .
$$

Hence there is a sequence of canonical morphisms:

$$
\begin{aligned}
R \rho_{!} v_{N N}(F) & \longrightarrow l ! R l_{!} R \rho_{!} v_{N N}(F) \simeq l^{!} R(T g)_{!} v_{N N}(F) \\
& \longrightarrow l v_{N M}(F) .
\end{aligned}
$$

Since $v_{N N}(F)$ coincides with $v_{N}(F)$ by Proposition 3.2, we have constructed a canonical morphism:

$$
R \rho_{!} v_{N}(F) \longrightarrow l^{!} v_{N M}(F)
$$

To verify that it is in fact an isomorphism, it is enough to show the following lemma.

Lemma 3.11. Assume (3.21) and (3.22), and let $\left(v^{\prime} \frac{\partial}{\partial x^{\prime}}, v^{\prime \prime} \frac{\partial}{\partial x^{\prime \prime}}, x^{\prime \prime \prime}\right)$ be a coordinate system of $T_{N} M \times{ }_{M} T_{M} X$. Then, for $p_{0}=\left(v_{0}^{\prime}, 0,0\right) \in N \times{ }_{M} T_{M} X$ and for every $j \in \mathbb{Z}$,

$$
H^{j}\left[R \Gamma_{\left(N \times{ }_{M} T_{M} X\right)}\left(v_{N M}(F)\right)\right]_{p_{0}} \stackrel{\sim}{\sim} \lim _{Z, U} H_{Z \cap U}^{j}(U ; F),
$$

where

$$
Z=\left\{\left|x^{\prime}\right| \geq \varepsilon\left|x^{\prime \prime}\right|\right\}, U=\left(U^{\prime} \times X^{\prime \prime} \times X^{\prime \prime}\right) \cap\{|x|<\varepsilon\},
$$

for open cones $U^{\prime}$ of $X_{x^{\prime}}^{\prime}$ such that $v_{0}^{\prime} \in U^{\prime}$ and $\varepsilon>0$.

Proof. Fix an open cone $U^{\prime} \subset X_{x^{\prime}}^{\prime}$ such that $v_{0}^{\prime} \in U^{\prime}$ and $\varepsilon>0$, and set:

$$
\left\{\begin{array}{l}
U:=\left(U^{\prime} \times X^{\prime \prime} \times X^{\prime \prime}\right) \cap\{|x|<\varepsilon\}, \\
\tilde{U}:=U \cap\left\{\left|x^{\prime}\right|<\varepsilon\left|x^{\prime \prime}\right|\right\}, \\
V:=U^{\prime} \times X_{v^{\prime \prime}}^{\prime \prime} \times\left\{\left|x^{\prime \prime \prime}\right|<\varepsilon\right\}, \\
\tilde{V}:=U^{\prime} \times\left(X_{v^{\prime \prime}}^{\prime \prime}-\{0\}\right) \times\left\{\left|x^{\prime \prime \prime}\right|<\varepsilon\right\},
\end{array}\right.
$$

where $V$ and $\tilde{V}$ are biconic open cones of $T_{N} M \underset{M}{\times} T_{M} X$ of the producttype. Then by Proposition 2.7,

$$
C_{N M}(X \backslash U) \cap V=\varnothing \quad \text { and } \quad C_{N M}(X \backslash \tilde{U}) \cap \tilde{V}=\emptyset .
$$

Applying Proposition 2.9 (ii), we can construct a natural morphism:

$$
R \Gamma(U ; F) \longrightarrow R \Gamma\left(U ; R \tilde{p}_{*} \tilde{p}^{-1} F\right)
$$




$$
\begin{aligned}
& \simeq R \Gamma\left(\tilde{p}^{-1}(U) ; \tilde{p}^{-1} F\right) \\
& \longrightarrow R \Gamma\left(V ; s^{-1} R j_{*} \tilde{p}^{-1} F\right) \\
& \simeq R \Gamma\left(V ; v_{N M}(F)\right)
\end{aligned}
$$

and similarly we have:

$$
R \Gamma(\tilde{U} ; F) \longrightarrow R \Gamma\left(\tilde{V} ; v_{N M}(F)\right) .
$$

Notice that

$$
\overline{\tilde{p}^{-1}(U \backslash \tilde{U})} \cap V \subset(V \backslash \tilde{V})=U^{\prime} \times\{0\} \times\left\{\left|x^{\prime \prime \prime}\right|<\varepsilon\right\}
$$

and there exists a natural morphism:

$$
R \Gamma_{(U \backslash \tilde{U})}(U ; F) \longrightarrow R \Gamma_{(V \backslash \tilde{V})}(V ; F) .
$$

Now we have a morphism of distinguished triangles:

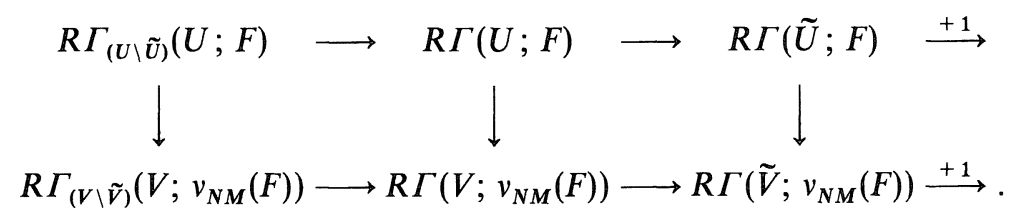

If we shrink $U, \tilde{U}, V$ and $\tilde{V}$ by replacing $U^{\prime}$ and $\varepsilon>0$, we obtain:

$$
\left\{\begin{array}{l}
\frac{\lim _{U}}{U} H^{j}(U ; F) \stackrel{\sim}{\longrightarrow} \underset{V}{\lim _{V}} H^{j}\left(V ; v_{N M}(F)\right) \\
\underset{\tilde{U}}{\lim ^{j}}(\tilde{U} ; F) \stackrel{\sim}{\longrightarrow} \underset{\tilde{V}}{\lim } H^{j}\left(\tilde{V} ; v_{N M}(F)\right),
\end{array}\right.
$$

by Theorem 3.8 (ii) and Proposition 2.7. Applying the five lemma to (3.60), we have:

$$
\begin{aligned}
\varliminf_{U, \tilde{U}} H_{(U \backslash \tilde{U})}^{j}(U ; F) & \stackrel{\sim}{\longrightarrow} \varliminf_{V, \tilde{V}} H_{(V \backslash \tilde{V})}^{j}\left(V ; v_{N M}(F)\right) \\
& \simeq H^{j}\left[R \Gamma_{\left(N_{M} T_{M} X\right)}\left(v_{N M}(F)\right)\right]_{p_{0}}
\end{aligned}
$$

Hence the proof is complete.

The method of the proof of the above Lemma allows us to obtain the following result:

Proposition 3.12. Assume (3.21) and (3.22), and set:

$$
T_{N} M \underset{M}{\times} T_{M}^{*} X=X_{\xi^{\prime}}^{\prime} \times X_{v^{\prime \prime}}^{\prime \prime} \times X_{x^{\prime \prime \prime}}^{\prime \prime \prime}
$$


Then for $p_{0}=\left(\xi_{0}^{\prime}, v_{0}^{\prime \prime}, 0\right) \in \dot{T}_{N} M \times{ }_{M} \dot{T}_{M}^{*} X$ and every $j \in \mathbb{Z}$, we have:

$$
\varliminf_{A} H^{j}\left[R \Gamma_{A}\left(v_{N M}(F)\right)\right]_{\left(0, v_{0}^{\prime \prime}, 0\right)} \stackrel{\sim}{\sim} \lim _{Z, U} H_{Z \cap U}^{j}(U ; F),
$$

where $A=A^{\prime} \times X^{\prime \prime} \times X^{\prime \prime \prime}, A^{\prime}$ ranges through the family of closed convex cones of $X^{\prime}$ such that $\left(A^{\prime}-\{0\}\right) \subset\left\{x^{\prime} \in X^{\prime} \mid\left\langle x^{\prime}, \xi_{0}^{\prime}\right\rangle>0\right\}$,

$$
\left\{\begin{array}{l}
Z=A^{\prime} \times X^{\prime \prime} \times X^{\prime \prime \prime} \\
U=\left(X^{\prime} \times U^{\prime \prime} \times X^{\prime \prime \prime}\right) \cap\left\{\left|x^{\prime}\right|<\varepsilon\left|x^{\prime \prime}\right|,|x|<\varepsilon\right\}, \varepsilon>0,
\end{array}\right.
$$

and $U^{\prime \prime}$ ranges through the family of open cones of $X^{\prime \prime}$ such that $v_{0}^{\prime \prime} \in U^{\prime \prime}$.

Proof. Similar to that of Lemma 3.11, and we shall omit it.

Denote by $\tau_{M}$ and $\tau_{N}$ the projection:

$$
T_{N} M \stackrel{\tau_{M}}{\longleftarrow} T_{N} M \underset{M}{\times} T_{M} X \stackrel{\tau_{N}}{\longrightarrow} N \underset{M}{\times} T_{M} X,
$$

and let us summarize the results of Proposition 3.2, Theorem 3.7, Theorem 3.9 and Theorem 3.10.

Theorem 3.13. Let $F \in \mathbf{D}^{\mathrm{b}}(X)$. Then there are canonical isomorphisms:

$$
\begin{aligned}
& \left.R \tau_{N *} v_{N M}(F) \stackrel{\sim}{\sim} v_{M}(F)\right|_{N_{M} T_{M} X}, \\
& \left.R \tau_{M *} v_{N M}(F) \stackrel{\sim}{\sim} v_{N}(F)\right|_{T_{N} M}, \\
& R \tau_{N !} v_{N M}(F) \stackrel{\sim}{\longleftarrow} R \rho_{!} v_{N}(F), \\
& R \tau_{M !} v_{N M}(F) \stackrel{\sim}{\longleftarrow} v_{N} R \Gamma_{M}(F),
\end{aligned}
$$

where $\rho$ denotes the projection $T_{N} X \rightarrow N \underset{M}{\times} T_{M} X$.

\section{§4. Bimicrolocalization Functors}

\subsection{Functors $v \mu_{N M}$ and $\mu_{N M}$}

Let

$$
\mathscr{F}_{1}: \mathbf{D}^{\mathrm{b}}\left(T_{N} M \underset{M}{\times} T_{M} X\right) \longrightarrow \mathbf{D}^{\mathrm{b}}\left(T_{N} M \underset{M}{\times} T_{M}^{*} X\right)
$$

and 


$$
\mathscr{F}_{2}: \mathbf{D}^{\mathrm{b}}\left(T_{N} M \underset{M}{\times} T_{M}^{*} X\right) \longrightarrow \mathbf{D}^{\mathrm{b}}\left(T_{N}^{*} M \underset{M}{\times} T_{M}^{*} X\right)
$$

be Fourier-Sato transformations on $T_{M} X$ and $T_{N} M$ respectively. We shall use the projections:

$$
T_{N} M \stackrel{\pi_{M}}{\longleftarrow} T_{N} M \underset{M}{\times} T_{M}^{*} X \stackrel{\tau_{N}}{\longrightarrow} N \underset{M}{\times} T_{M}^{*} X,
$$

and

$$
T_{N}^{*} M \stackrel{\pi_{M}}{\longleftarrow} T_{N}^{*} M \underset{M}{\times} T_{M}^{*} X \stackrel{\pi_{N}}{\longrightarrow} N \underset{M}{\times} T_{M}^{*} X,
$$

Definition 4.1. For $F \in \mathbf{D}^{\mathrm{b}}(X)$, set:

$$
\begin{aligned}
& v \mu_{N M}(F):=\mathscr{F}_{1}\left(v_{N M}(F)\right) \in \mathbf{D}^{\mathrm{b}}\left(T_{N} M \underset{M}{\times} T_{M}^{*} X\right), \\
& \mu_{N M}(F):=\mathscr{F}_{2}\left(v \mu_{N M}(F)\right) \in \mathbf{D}^{\mathrm{b}}\left(T_{N}^{*} M \underset{M}{\times} T_{M}^{*} X\right) .
\end{aligned}
$$

We leave the construction of the functor $\mu v_{N M}(F)$ to the reader.

Theorem 4.2. Let $F \in \mathbf{D}^{\mathrm{b}}(X)$. Then:

(i) $v \mu_{N M}(F)$ is a biconic object of $\mathbf{D}^{\mathrm{b}}\left(T_{N} M \underset{M}{\times} T_{M}^{*} X\right)$.

(ii) In the situation of (3.21) and (3.22), let $\left(\xi^{\prime} d x^{\prime}, v^{\prime \prime} \frac{\partial}{\partial x^{\prime \prime}}, x^{\prime \prime \prime}\right)$ be the coordinates of $T_{N} M \times_{M} T_{M}^{*} X$. Let $p_{0}=\left(\xi_{0}^{\prime} d x^{\prime}, v_{0}^{\prime \prime} \frac{\partial}{\partial x^{\prime \prime}}, 0\right) \in \dot{T}_{N} M \times_{M} \dot{T}_{M}^{*} X$. Then for every $j \in \mathbb{Z}$ :

$$
H^{j}\left[v \mu_{N M}(F)\right]_{p_{0}}=\lim _{Z, U} H_{Z \cap U}^{j}(U ; F),
$$

where the inductive limit is taken over the family of subsets $Z$ and $U$ of $X$ such that: $Z=\gamma^{\prime} \times X_{x^{\prime \prime}}^{\prime \prime} \times X_{x^{\prime \prime \prime}}^{\prime \prime \prime}, \gamma^{\prime}$ is a closed cone of $X_{x^{\prime}}^{\prime}$, such that

$$
\begin{aligned}
\left(\gamma^{\prime} \backslash\{0\}\right) & \subset\left\{x^{\prime} \in X^{\prime} ;\left\langle x^{\prime}, \xi_{0}^{\prime}\right\rangle>0\right\}, \\
U=\left(X_{x^{\prime}}^{\prime} \times U^{\prime \prime} \times X_{x^{\prime \prime \prime}}^{\prime \prime \prime}\right) & \cap\left\{\left|x^{\prime}\right|<\varepsilon\left|x^{\prime \prime}\right|,|x|<\varepsilon\right\}, \quad \varepsilon>0,
\end{aligned}
$$

and $U^{\prime \prime}$ is an open cone of $X_{x^{\prime \prime}}^{\prime \prime}$ such that $v_{0}^{\prime \prime} \in U^{\prime \prime}$.

(iii) We have canonical isomorphisms:

$$
\begin{aligned}
& \left.R \tau_{N *} v \mu_{N M}(F) \stackrel{\sim}{\sim} \mu_{M}(F)\right|_{N \times T_{M}^{*} X}, \\
& R \pi_{M *} v \mu_{N M}(F) \stackrel{\sim}{\longleftarrow} v_{N} R \Gamma_{M}(F), \\
& \left.R \tau_{N !} v \mu_{N M}(F) \stackrel{\sim}{\longleftarrow} \mu_{N}(F)\right|_{N_{M} \times T_{M}^{*} X},
\end{aligned}
$$




$$
\left.R \pi_{M !} v \mu_{N M}(F) \stackrel{\sim}{\sim} v_{N}(F)\right|_{T_{N} M} \otimes \omega_{M \backslash X} .
$$

Proof. (i) Obvious.

(ii) This is nothing but Proposition 3.12.

(iii) Using Proposition 3.7.13 and Proposition 3.7.14 of [11], these formulas are immediate consequences of Theorem 3.13.

Theorem 4.3. Let $F \in \mathbf{D}^{\mathrm{b}}(X)$. Then:

(i) $\mu_{N M}(F)$ is a biconic object of $\mathbf{D}^{\mathbf{b}}\left(T_{N}^{*} M \underset{M}{\times} T_{M}^{*} X\right)$.

(ii) In the situation of (3.21) and (3.22), let $\left(\xi^{\prime} d x^{\prime}, \xi^{\prime \prime} d x^{\prime \prime}, x^{\prime \prime \prime}\right)$ be the coordinates of $T_{N}^{*} M \times{ }_{M} T_{M}^{*} X$. Let $q_{0}=\left(\xi_{0}^{\prime} d x^{\prime}, \xi_{0}^{\prime \prime} d x^{\prime \prime}, 0\right) \in \dot{T}_{N}^{*} M \times{ }_{M} \dot{T}_{M}^{*} X$. Then for every $j \in \mathbb{Z}$ :

$$
H^{j}\left[\mu_{N M}(F)\right]_{q_{0}}=\varliminf_{Z, U} H_{Z \cap U}^{j}(U ; F),
$$

where the inductive limit is taken over the family of subsets $Z$ and $U$ of $X$ such that: $Z=\left\{\left(\{0\} \times \gamma^{\prime \prime}\right)+\gamma\right\} \times X_{x^{\prime \prime \prime}}^{\prime \prime \prime}, \gamma^{\prime \prime} \subset X_{x^{\prime \prime}}^{\prime \prime}$ and $\gamma \subset X_{x^{\prime}}^{\prime} \times X_{x^{\prime \prime}}^{\prime \prime}$ are closed cones such that

$$
\begin{gathered}
\left(\gamma^{\prime \prime} \backslash\{0\}\right) \subset\left\{x^{\prime \prime} ;\left\langle x^{\prime \prime}, \xi_{0}^{\prime \prime}\right\rangle>0\right\}, \\
(\gamma \backslash\{0\}) \subset\left\{\left(x^{\prime}, x^{\prime \prime}\right) ;\left\langle x^{\prime}, \xi_{0}^{\prime}\right\rangle>0\right\},
\end{gathered}
$$

and $U=\{x \in X ;|x|<\varepsilon\}$ for some $\varepsilon>0$.

(iii) We have canonical isomorphisms:

$$
\begin{aligned}
& \left.R \pi_{N *} \mu_{N M}(F) \stackrel{\sim}{\sim} \mu_{N}(F)\right|_{N_{M} \times T_{M}^{*} X}, \\
& R \pi_{M *} \mu_{N M}(F) \stackrel{\sim}{\sim} \mu_{N} R \Gamma_{M}(F), \\
& \left.R \pi_{N !} \mu_{N M}(F) \stackrel{\sim}{\sim} \mu_{M}(F)\right|_{N_{M} \times T_{M}^{*} X} \otimes \omega_{N / M}, \\
& R \pi_{M !} \mu_{N M}(F) \stackrel{\sim}{\sim} R \theta_{!} \mu_{N}(F),
\end{aligned}
$$

where $\theta$ denotes the projection $T_{N}^{*} X \rightarrow T_{N}^{*} M$.

Proof. (i) is obvious.

(iii) follows from Theorem 4.2 (iii) by using Proposition 3.7 .13 and Proposition 3.7 .14 of [11]. It remains to show (ii).

(ii) Assume $x^{\prime}=\left(x_{1}^{\prime}, \ldots, x_{n^{\prime}}^{\prime}\right), x^{\prime \prime}=\left(x_{1}^{\prime \prime}, \ldots, x_{n^{\prime \prime}}^{\prime \prime}\right), \xi_{0}^{\prime} d x^{\prime}=d x_{1}^{\prime}$, and $\xi_{0}^{\prime \prime} d x^{\prime \prime}=$ $d x_{1}^{\prime \prime}$. Now set for $\varepsilon>0$ : 


$$
\left\{\begin{array}{l}
U_{\varepsilon}:=\left\{\left(x^{\prime}, x^{\prime \prime}, x^{\prime \prime \prime}\right) \in X ;\left|x^{\prime}\right|<\varepsilon^{2},\left|x^{\prime \prime}\right|<\varepsilon,\left|x^{\prime \prime \prime}\right|<\varepsilon\right\}, \\
\hat{U}_{\varepsilon}:=U_{\varepsilon} \cap\left\{\left(x^{\prime}, x^{\prime \prime}, x^{\prime \prime \prime}\right) \in X ;\left|x^{\prime}\right|<\varepsilon\left|x^{\prime \prime}\right|, x_{1}^{\prime \prime}<\varepsilon\left|\left(x_{2}^{\prime \prime}, \ldots, x_{n^{\prime \prime}}^{\prime \prime}\right)\right|\right\} \\
\hat{Z}_{\varepsilon}:=\left\{\left(x^{\prime}, x^{\prime \prime}, x^{\prime \prime \prime}\right) \in X ; x_{1}^{\prime} \geq \varepsilon\left|\left(x_{2}^{\prime}, \ldots, x_{n^{\prime}}^{\prime}\right)\right|\right\} .
\end{array}\right.
$$

Since $T_{N} M \times{ }_{M} T_{M} X=\mathbb{R}_{v^{\prime}}^{n^{\prime}} \times \mathbb{R}_{v^{\prime \prime}}^{n^{\prime \prime}} \times \mathbb{R}_{x^{\prime \prime \prime}}^{n^{\prime \prime \prime}}$, with the notations $v^{\prime}=\left(v_{1}^{\prime}, \ldots, v_{n^{\prime}}^{\prime}\right)$ and $v^{\prime \prime}=\left(v_{1}^{\prime \prime}, \ldots, v_{n^{\prime \prime}}^{\prime \prime}\right)$, we can define following subsets of $T_{N} M \times{ }_{M} T_{M} X$ for $\varepsilon>0$ :

$$
\left\{\begin{array}{l}
V_{\varepsilon}:=\left\{\left(v^{\prime}, v^{\prime \prime}, x^{\prime \prime \prime}\right) ;\left|x^{\prime \prime \prime}\right|<\varepsilon\right\}, \\
\hat{V}_{\varepsilon}:=\left\{\left(v^{\prime}, v^{\prime \prime}, x^{\prime \prime \prime}\right) ;\left|x^{\prime \prime \prime}\right|<\varepsilon, v_{1}^{\prime \prime}<\varepsilon\left|\left(v_{2}^{\prime \prime}, \ldots, v_{n^{\prime \prime}}^{\prime \prime}\right)\right|\right\}, \\
\hat{A}_{\varepsilon}:=\left\{\left(v^{\prime}, v^{\prime \prime}, x^{\prime \prime \prime}\right) ; v_{1}^{\prime} \geq \varepsilon\left|\left(v_{2}^{\prime}, \ldots, v_{n^{\prime}}^{\prime}\right)\right|\right\} .
\end{array}\right.
$$

Then, by the techniques used in the proof of Lemma 3.11, we have for every $j \in \mathbb{Z}$ :

$$
\begin{aligned}
& \varliminf_{\varepsilon>0} H_{\left(\hat{A}_{\varepsilon} \cap V_{\varepsilon}\right)}^{j}\left(V_{\varepsilon} ; v_{N M}(F)\right) \stackrel{\sim}{\sim} \varliminf_{\varepsilon>0} H_{\left(\hat{Z}_{\varepsilon} \cap U_{\varepsilon}\right)}^{j}\left(U_{\varepsilon} ; F\right), \\
& \varliminf_{\varepsilon>0} H_{\left(\hat{A}_{\varepsilon} \cap \hat{V}_{\varepsilon}\right)}^{j}\left(\hat{V}_{\varepsilon} ; v_{N M}(F)\right) \stackrel{\sim}{\sim} \varliminf_{\varepsilon>0} H_{\left(\hat{Z}_{\varepsilon} \cap \hat{U}_{\varepsilon}\right)}^{j}\left(\hat{U}_{\varepsilon} ; F\right) .
\end{aligned}
$$

Now again, set for every $\varepsilon>0$ :

$$
\left\{\begin{array}{l}
Z_{\varepsilon}:=\left(\hat{Z}_{\varepsilon} \cap U_{\varepsilon}\right) \backslash \hat{U}_{\varepsilon}, \\
A_{\varepsilon}:=\left(\hat{A}_{\varepsilon} \cap V_{\varepsilon}\right) \backslash \hat{V}_{\varepsilon}
\end{array}\right.
$$

Then one has

$$
C_{N M}\left(Z_{\varepsilon}\right) \cap V_{\varepsilon} \subset A_{\varepsilon}
$$

by Proposition 2.6 (ii), and there exist a canonical morphism:

$$
R \Gamma_{Z_{\varepsilon}}\left(U_{\varepsilon} ; F\right) \longrightarrow R \Gamma_{A_{\varepsilon}}\left(V_{\varepsilon} ; v_{N M}(F)\right)
$$

and a morphism between distinguished triangles:

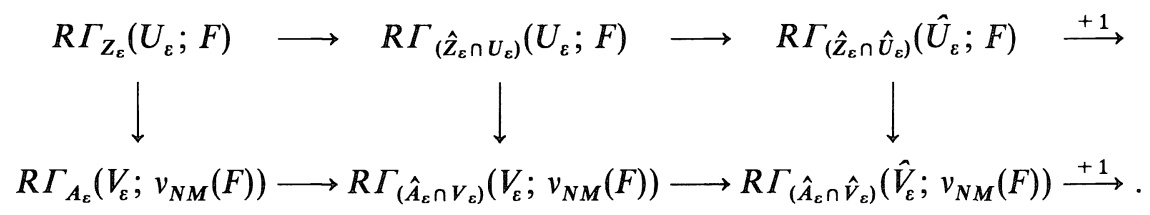

By virtue of (4.20), (4.21) and the five Lemma, we have for every $j \in \mathbb{Z}$ :

$$
\varliminf_{\varepsilon>0} H_{A_{\varepsilon}}^{j}\left(V_{\varepsilon} ; v_{N M}(F)\right) \stackrel{\sim}{\sim} \varliminf_{\varepsilon>0} H_{Z_{\varepsilon}}^{j}\left(U_{\varepsilon} ; F\right),
$$

where the left hand side coincides with $H^{j}\left[\mu_{N M}(F)\right]_{q_{0}}\left(q_{0}=\left(d x_{1}^{\prime}, d x_{1}^{\prime \prime}, 0\right)\right)$. Now notice: 
(4.27)

$$
\begin{aligned}
Z_{\varepsilon}= & U_{\varepsilon} \cap\left\{\left(x^{\prime}, x^{\prime \prime}, x^{\prime \prime \prime}\right) ; x_{1}^{\prime} \geq \varepsilon\left|\left(x_{2}^{\prime}, \ldots, x_{n^{\prime}}^{\prime}\right)\right|\right\} \\
& \cap\left[\left\{\left(x^{\prime}, x^{\prime \prime}, x^{\prime \prime}\right) ; x_{1}^{\prime \prime} \geq \varepsilon\left|\left(x_{2}^{\prime \prime}, \ldots, x_{n^{\prime \prime}}^{\prime \prime}\right)\right|\right\} \cup\left\{\left(x^{\prime}, x^{\prime \prime}, x^{\prime \prime \prime}\right) ;\left|x^{\prime}\right| \geq \varepsilon\left|x^{\prime \prime}\right|\right\}\right],
\end{aligned}
$$

and it is a direct calculation that the right hand side of (4.26) coincides with the right hand side of (4.11). This completes the proof of the theorem.

\subsection{Functorial properties of $v \mu_{N M}$ and $\mu_{N M}$}

Let $f:\left(X^{\prime}, M^{\prime}, N^{\prime}\right) \rightarrow(X, M, N)$ be a morphism of triplets of manifolds. The morphism:

$$
T f: T_{N^{\prime}} M^{\prime} \underset{M^{\prime}}{\times} T_{M^{\prime}} X^{\prime} \longrightarrow T_{N} M \underset{M}{\times} T_{M} X
$$

induced by $f$ is decomposed in the following way:

$$
\begin{aligned}
T_{N^{\prime}} M^{\prime} \underset{M^{\prime}}{\times} T_{M^{\prime}} X^{\prime} & \underset{f_{1}}{\longrightarrow} T_{N^{\prime}} M^{\prime} \underset{M^{\prime}}{\times}\left(M^{\prime} \underset{M}{\times} T_{M} X\right) \\
& \simeq T_{N^{\prime}} M^{\prime} \underset{M}{\times} T_{M} X \\
& \underset{f_{2}}{\longrightarrow}\left(N^{\prime} \underset{N}{\times} T_{N} M\right) \underset{M}{\times} T_{M} X \\
& \underset{f_{3}}{\longrightarrow} T_{N} M \underset{M}{\times} T_{M} X .
\end{aligned}
$$

If we consider these morphisms w.r.t. the second vector bundle structure, $f_{1}$ is a morphism of vector bundles over $T_{N^{\prime}} M^{\prime}$ and $f_{2}$ and $f_{3}$ are base changes of vector bundles. Taking the dual of the above sequence of morphisms, we have:

$$
\begin{aligned}
T_{N^{\prime}} M^{\prime} \underset{M^{\prime}}{\times} T_{M^{\prime}}^{*} X^{\prime} \underset{{ }_{f_{f_{1}}}}{\stackrel{\longrightarrow}{\longrightarrow}} T_{N^{\prime}} M^{\prime} \underset{M}{\times} T_{M}^{*} X \\
\underset{f_{2 \pi}}{\stackrel{\longrightarrow}{\longrightarrow}} T_{N} M \underset{M}{\times}\left(N^{\prime} \underset{M}{\times} T_{M}^{*} X\right) \\
\underset{f_{3 \pi}}{\longrightarrow} T_{N} M \underset{M}{\times} T_{M}^{*} X .
\end{aligned}
$$

Next let us consider them w.r.t. the first vector bundle structure. Then ${ }^{t} f_{1}$ and $f_{3 \pi}$ are base changes of vector bundles and $f_{2 \pi}$ is a morphism of vector bundles over $N^{\prime} \underset{M}{\times} T_{M}^{*} X$. Taking the dual again, we have:

$$
T_{N^{\prime}}^{*} M^{\prime} \underset{M^{\prime}}{\times} T_{M^{\prime}}^{*} M^{\prime} \underset{{ }_{t} f_{1 \pi}}{\longleftarrow} T_{N^{\prime}}^{*} M^{\prime} \underset{M}{\times} T_{M}^{*} X
$$




$$
\begin{aligned}
& \underset{t_{f_{2 \pi}}}{\stackrel{N}{N}} T_{N}^{*} M \underset{M}{\times}\left(N^{\prime} \underset{M}{\times} T_{M}^{*} X\right) \\
& \underset{f_{3 \pi \pi}}{\longrightarrow} T_{N}^{*} M \underset{M}{\times} T_{M}^{*} X .
\end{aligned}
$$

The following propositions are very similar to the corresponding results in [11], and the proofs proceed completely similarly. Hence we shall omit them.

Proposition 4.4. Let $F \in \mathbf{D}^{\mathrm{b}}(X), G \in \mathbf{D}^{\mathrm{b}}\left(X^{\prime}\right)$. Then there are commutative diagrams below:

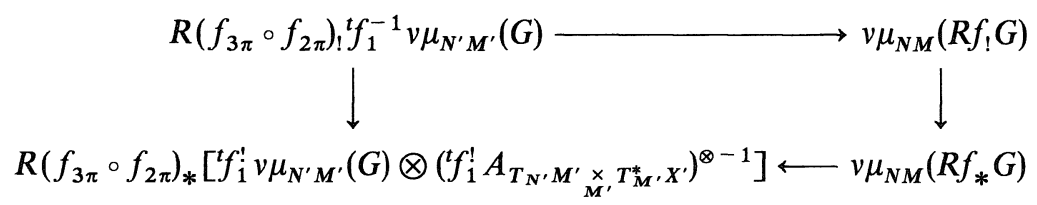

and

$R^{t} f_{1 !}\left[\left(f_{3 \pi} \circ f_{2 \pi}\right)^{!} A_{T_{N} M \times{ }_{M}{ }^{*}{ }_{M} X} \otimes\left(f_{3 \pi} \circ f_{2 \pi}\right)^{-1} v \mu_{N M}(F)\right] \longrightarrow v \mu_{N^{\prime} M^{\prime}}\left(f^{\prime} A_{X} \otimes f^{-1} F\right)$

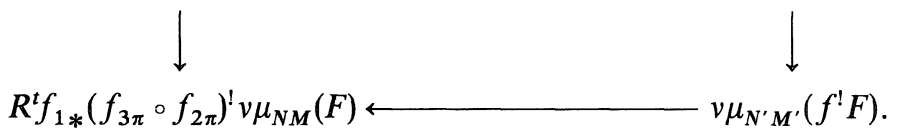

Proposition 4.5. Let $F \in \mathbf{D}^{\mathrm{b}}(X), G \in \mathbb{D}^{\mathrm{b}}\left(X^{\prime}\right)$. Then there are commutative diagrams below:

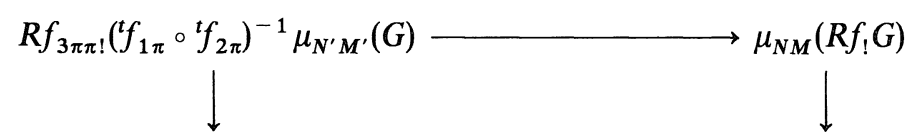

$$
R f_{3 \pi \pi *}\left[\left({ }^{t} f_{1 \pi} \circ{ }^{t} f_{2 \pi}\right)^{!} \mu_{N^{\prime} M^{\prime}}(G) \otimes\left\{\left({ }^{t} f_{1 \pi}{ }^{t} f_{2 \pi}\right)^{!} A_{T_{N^{\prime} M^{\prime} \times M^{\prime}} T_{M^{\prime}}^{*} X^{\prime}}\right\}^{\otimes-1}\right] \longleftarrow \mu_{N M}\left(R f_{*} G\right)
$$

and

$$
R\left({ }^{t} f_{1 \pi}{ }^{t} f_{2 \pi}\right)_{!}\left[f_{3 \pi \pi}^{!} A_{T_{N}^{*} M \times T_{M}^{*} X} \otimes f_{3 \pi \pi}^{-1} \mu_{N M}(F)\right] \longrightarrow \mu_{N^{\prime} M^{\prime}}\left(f^{!} A_{X} \otimes f^{-1} F\right)
$$

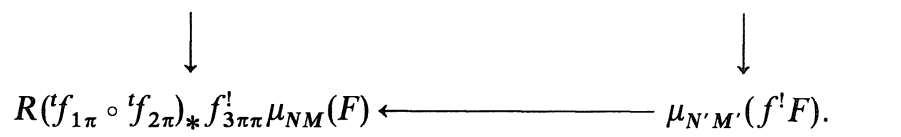

Proposition 4.6. Let $F \in \mathbb{D}^{\mathrm{b}}(X), G \in \mathbf{D}^{\mathrm{b}}\left(X^{\prime}\right)$. Then, we have canonical morphisms:

$$
v \mu_{N M}(F) \bigotimes^{L} v \mu_{N^{\prime} M^{\prime}}(G) \longrightarrow v \mu_{\left(N \times N^{\prime}\right)\left(M \times M^{\prime}\right)}\left(F \bigotimes^{L} G\right)
$$

and

$$
\mu_{N M}(F) \bigotimes^{L} \mu_{N^{\prime} M^{\prime}}(G) \longrightarrow \mu_{\left(N \times N^{\prime}\right)\left(M \times M^{\prime}\right)}\left(F \bigotimes^{L} G\right) .
$$


Now let us consider the natural morphisms given by the addition of covectors :

$$
\gamma:\left(T_{N} M \underset{M}{\times} T_{M}^{*} X\right) \underset{T_{N} M}{\times}\left(T_{N} M \underset{M}{\times} T_{M}^{*} X\right) \longrightarrow T_{N} M \underset{M}{\times} T_{M}^{*} X
$$

and

$$
\theta:\left(T_{N}^{*} M \underset{M}{\times} T_{M}^{*} X\right) \underset{N}{\times}\left(T_{N}^{*} M \underset{M}{\times} T_{M}^{*} X\right) \longrightarrow T_{N}^{*} M \underset{M}{\times} T_{M}^{*} X .
$$

Then we have:

Proposition 4.7. Let $F, G \in \mathbf{D}^{\mathrm{b}}(X)$. There are canonical morphisms of "product":

$$
R \gamma_{!}\left[v \mu_{N M}(F) \bigotimes_{T_{N} M}^{L} v \mu_{N M}(G)\right] \longrightarrow v \mu_{N M}\left(F \otimes{ }^{L} G\right) \otimes \omega_{M / X}
$$

and

$$
R \theta_{!}\left[\mu_{N M}(F) \bigotimes_{N}^{L} \mu_{N M}(G)\right] \longrightarrow \mu_{N M}\left(F \otimes L{ }^{L} G\right) \otimes \omega_{N / X}
$$

\subsection{Various generalizations of the functor $\mu h o m$}

Using the diagonal embedding, the functor of Sato's microlocalization allows one to define the bifunctor $\mu$ hom $(\cdot, \cdot)([11])$. Here we shall proceed similarly, using the functor of second microlocalization, in various geometrical situations. The results in this section were obtained during some discussions with P. Schapira. In particular, the idea to work in the relative setting is due to him.

First, consider a smooth morphism of manifolds $f: X \rightarrow S$. We identify $X \times{ }_{S} T^{*} S$ to an involutive submanifold of $T^{*} X$ that we denote by $V$ :

$$
V:=X \times{ }_{S} T^{*} S,
$$

and one defines as usual the relative cotangent bundle $T^{*}(X / S)$ by the exact sequence of vector bundles:

$$
0 \longrightarrow X \times{ }_{S} T^{*} S \longrightarrow T^{*} X \longrightarrow T^{*}(X / S) \longrightarrow 0 .
$$

Set $L:=X \times{ }_{S} X$ and consider the triplet of manifolds:

$$
X \simeq \Delta_{X} \hookrightarrow L:=X \times{ }_{S} X \subset X \times X .
$$

The first projection from $T^{*}(X \times X)$ to $T^{*} X$ defines the isomorphisms:

$$
\begin{aligned}
T_{\Delta_{X}}^{*}(X \times X) & \simeq T^{*} X, \\
\Delta_{X} \times{ }_{L} T_{L}^{*}(X \times X) & \simeq X \times{ }_{S} T^{*} S .
\end{aligned}
$$


Moreover, the natural morphism $T_{\Delta_{X}}^{*}(X \times X) \rightarrow T_{\Delta_{X}}^{*}\left(X \times_{S} X\right)$ being zero on $X \times{ }_{S} T^{*} S$, it defines the morphism:

$$
T^{*}(X / S) \longrightarrow T_{\Delta_{X}}^{*}\left(X \times_{S} X\right)=T_{\Delta x}^{*} L
$$

and one checks immediately that this is an isomorphism. Summarizing, we have got a natural isomorphism:

$$
\begin{aligned}
T_{\Delta_{X}}^{*} L \times{ }_{L} T_{L}^{*}(X \times X) & \simeq T^{*}(X / S) \times{ }_{S} T^{*} S \\
& \simeq T^{*}(X / S) \times{ }_{X} V .
\end{aligned}
$$

The canonical projection $T^{*} X \rightarrow X$ and the Hamiltonian isomorphism define a sequence of morphisms:

$$
\begin{aligned}
V \times_{X} T^{*} X & \longrightarrow V \times_{\left(T^{*} M\right)} T^{*}\left(T^{*} X\right) \\
\simeq V \times_{\left(T^{*} X\right)} T\left(T^{*} X\right) & \longrightarrow T_{V}\left(T^{*} X\right) .
\end{aligned}
$$

The composition of these morphisms sends $V \times{ }_{X}\left(X \times{ }_{S} T^{*} S\right)$ to the zero-section of $T_{V}\left(T^{*} X\right)$ and we obtain a morphism:

$$
V \times{ }_{X} T^{*}(X / S) \longrightarrow T_{V}\left(T^{*} X\right)
$$

and one immediately checks, using local coordinates, that this morphism is an isomorphism.

Denote as usual by $q_{1}$ and $q_{2}$ the first and second projections from $X \times X$ to $X$.

Definition 4.8. Let $F$ and $G$ belong to $\mathbf{D}^{\mathrm{b}}(X)$. We set:

$$
\mu h o m_{f}(G, F)=\mu_{\Delta_{x} L} R \mathscr{H} \circ \operatorname{m}\left(q_{2}^{-1} G, q_{1}^{!} F\right) \text {. }
$$

Hence $\mu$ hom $m_{f}(G, F)$ is a biconic object on $T_{\Delta_{X}}^{*} L \times_{L} T_{L}^{*}(X \times X) \simeq T^{*}(X / S) \times_{X}$ $V \simeq T_{V}\left(T^{*} X\right)$. When restricting to $V$, we find:

$$
\left.\left.\mu h o m_{f}(G, F)\right|_{V} \simeq \mu h o m(G, F)\right|_{V}
$$

and when restricting to $T^{*}(X / S)$, we find a sheaf which coincides with the "relative $\mu$ hom-sheaf" of Ishimura [3].

It is possible to extend the previous construction to the case where $f$ is no more smooth by decomposing $f$ by the graph embedding, $f=p \circ s$, with $s: X \hookrightarrow X \times S, p: X \times S \rightarrow S$, and setting:

$$
\mu h o m_{f}(G, F)=\mu h o m_{p}\left(s_{*} G, s_{*} F\right) .
$$

Of course, one should prove that if $f$ is smooth, one recovers the previous definition. This is left to the reader. (Notice that a similar trick already appears in [21].)

Next, consider the case of a closed embedding: $f: N \hookrightarrow X$. Set 
$V:=N \times{ }_{X} T^{*} X$, and consider the triplet of manifolds :

$$
N \subset X \simeq \Delta_{X} \subset X \times X
$$

The projection $\left(T^{*} X, V\right) \rightarrow(X, N)$ defines $T_{V}\left(T^{*} X\right) \rightarrow T_{N} X$. Combining with the projection $T_{V}\left(T^{*} X\right) \rightarrow V$, we find the isomorphism:

$$
T_{V}\left(T^{*} X\right) \simeq T_{N} X \times_{X} V \simeq T_{N} X \times_{X} T^{*} X .
$$

Definition 4.9. Let $F$ and $G$ belong to $\mathbb{D}^{\mathrm{b}}(X)$. We set:

$$
\mu h o m_{f}(G, F)=v \mu_{N \Delta x} R \mathscr{H} \text { om }\left(q_{2}^{-1} G, q_{1}^{!} F\right) .
$$

Hence, $\mu h o m_{f}(G, F)$ is a biconic object on $T_{N} X \times_{X} T^{*} X \simeq T_{V}\left(T^{*} X\right)$. When restricting to $V$, we find:

$$
\left.\left.\mu h a m_{f}(G, F)\right|_{V} \simeq \mu h a m(G, F)\right|_{V},
$$

and when restricting to $T_{N} X$, we find

$$
\left.\mu h o m_{f}(G, F)\right|_{T_{N} X} \simeq v_{N} R \mathscr{H} \text { om }(G, F) .
$$

Here again, one could release the hypothesis that $f$ is an embedding by decomposing $f$ as $f=p \circ s$, with $s: N \hookrightarrow N \times X, p: N \times X \rightarrow X$, and setting:

$$
\mu h o m_{f}(G, F)=\mu h o m_{s}\left(p^{-1} G, p^{-1} F\right) .
$$

Remark 4.10. Let us consider a geometrical construction which often appears when dealing with involutive submanifolds. Let $f: Y \rightarrow X$ be a smooth morphism, let $g: S \rightarrow Y$ be a closed embedding, let $U$ be an open subset of $T^{*} Y$ and assume the intersection of $W:=U \cap\left(S \times{ }_{Y} T^{*} Y\right)$ and $Y \times_{X} T^{*} X$ is clean and $f_{\pi}$ induces an isomorphism:

$$
V^{\prime}:=U \cap\left(S \times{ }_{Y} T^{*} Y\right) \cap\left(Y \times{ }_{X} T^{*} X\right) \simeq V,
$$

where $V$ is an involutive submanifold of $T^{*} X$. Denote by $\rho$ and $\varpi$ the natural morphisms from $T_{V^{\prime}}\left(Y \times_{X} T^{*} X\right)$ to $T_{W} T^{*} Y$ and $T_{V} T^{*} X$ respectively. Let $F$ and $G$ be two objects of $\mathbb{D}^{\mathrm{b}}(X)$. Then it would be interesting to construct an object $\mu$ hom $m_{V}(G, F)$ such that:

$$
\varpi^{-1} \mu h \mathrm{om}_{V}(G, F) \simeq \rho^{-1} \mu h o m_{g}\left(f^{-1} G, f^{-1} F\right) .
$$

\section{\$5. Applications}

\section{H. Second microfunctions associated to a submanifold}

Let $M$ be a real analytic manifold of dimension $n$ and $N$ be a submanifold of $M$ of codimension $d$. We denote by $\mathcal{O}_{X}$ the sheaf of holomorphic functions on a complexification $X$ of $M$. Let us apply our theory to the triplet $(X, M, N)$. 
Definition 5.1. We set:

$$
\left\{\begin{array}{l}
\hat{\mathscr{C}}_{N M}:=v \mu_{N M}\left(\mathcal{O}_{X}\right) \otimes \text { or }_{M}[n], \\
\mathscr{C}_{N M}:=\mu_{N M}\left(\mathcal{O}_{X}\right) \otimes \text { or }_{N}[n] .
\end{array}\right.
$$

These are objects of the derived categories $\mathbf{D}^{\mathrm{b}}\left(T_{N} M \times{ }_{M} T_{M}^{*} X\right)$ and $\mathbf{D}^{\mathrm{b}}\left(T_{N}^{*} M \times{ }_{M}\right.$ $\left.T_{M}^{*} X\right)$ respectively.

Assume $M=\mathbb{R}^{n}, N=\{0\} \times \mathbb{R}_{x^{\prime \prime}}^{n-d}$ and $\Omega=\Omega^{\prime} \times \mathbb{R}_{x^{\prime \prime}}^{n-d} \subset M$ for an open convex proper cone $\Omega^{\prime}$ in $\mathbb{R}_{x^{\prime}}^{d}$, such that $(1,0, \ldots, 0) \in \Omega^{\prime}$, and take the associated coordinate system $\left(\sqrt{-1} \eta d x, v^{\prime} \frac{\partial}{\partial x^{\prime}}, x^{\prime \prime}\right)$ of $T_{N} M \times{ }_{M} T_{M}^{*} X$. Then we know by [24] and [25] that the complex $\hat{\mathscr{C}}_{N M}$ coincides with the inductive limit of the complex $\mathscr{C}_{\Omega \mid X}$ due to [20] in the following sense.

Theorem 5.2. The complex $\hat{\mathscr{C}}_{N M}$ is concentrated in degree 0 , and for $p_{0}:=\left(\frac{\partial}{\partial x_{1}}, x_{0}^{\prime \prime}\right) \in T_{N} M$ we have an isomorphism:

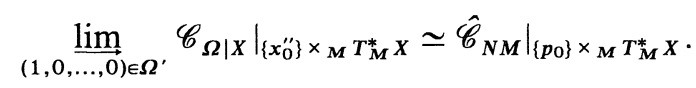

Now by virtue of Theorem 4.2 (iii), we can construct an infinitesimal version of the boundary value morphism. In fact consider the morphism:

$$
\hat{\mathscr{C}}_{N M} \longrightarrow \tau^{!} R \tau_{!}\left(\hat{\mathscr{C}}_{N M}\right) \simeq \tau^{-1}\left(\left.\mathscr{C}_{N \mid X}\right|_{N \times{ }_{M} T_{M}^{*} X}\right)[d],
$$

where $\tau: T_{N} M \times{ }_{M} T_{M}^{*} X \rightarrow N \times{ }_{M} T_{M}^{*} X$ denotes the canonical projection and $\mathscr{C}_{N \mid X}$ is the sheaf introduced in [19]. On account of the above theorem, we have constructed a morphism:

$$
\varliminf_{(1,0, \ldots, 0) \in \Omega^{\prime}} \mathscr{C}_{\Omega \mid X} \longrightarrow \mathscr{C}_{N \mid X}[d]
$$

on $N \times{ }_{M} T_{M}^{*} X$. Furthermore, let $\mathscr{M}$ be a coherent $\mathscr{D}_{X}$-module for which $N$ is non-characteristic. Then [24][25] shows the injectivity of the microlocal boundary value morphism:

$$
\left.H^{0} R \mathscr{H} \sigma_{m_{\mathscr{D}_{X}}}\left(\mathscr{M}, \hat{\mathscr{C}}_{N M}\right) \longrightarrow \tau^{-1} H^{0} R \mathscr{H}_{\sigma m_{\mathscr{D}_{X}}}\left(\mathscr{M}, \mathscr{C}_{N \mid X}[d]\right)\right|_{N \times{ }_{M} T_{M}^{*} X}
$$

Remark 5.3. It gives the uniqueness of microlocal boundary value problems and generalizes the result of Schapira [20] to higher codimensions. It can also be considered as a microlocal extension of Oaku's theorem in [16]. However we have not yet shown the injectivity by using the framework developed in this paper.

Finally let us consider the complex $\mathscr{C}_{N M}$ on $T_{N}^{*} M \times_{M} T_{M}^{*} X$. We can 
easily show by Theorem 4.3 (ii) and Kashiwara's abstract edge of the wedge theorem that it is concentrated in degree 0 . Let $\pi$ be the canonical projection $T_{N}^{*} M \times{ }_{M} T_{M}^{*} X \rightarrow N \times{ }_{M} T_{M}^{*} X$, and apply Sato's distinguished triangle $R \pi_{!} \rightarrow$ $R \pi_{*} \rightarrow R \dot{\pi}_{* \rightarrow 1}$ to the sheaf $\mathscr{C}_{N M}$. Then by Theorem 4.3 (iii) we get a distinguished triangle:

$$
\left.\left.\mathscr{C}_{M}\right|_{N \times{ }_{M} T_{M}^{*} X} \longrightarrow \mathscr{C}_{N \mid X}\right|_{N \times{ }_{M} T_{M}^{*} X}[d] \longrightarrow R \dot{\pi}_{*} \mathscr{C}_{N M}[d] \longrightarrow+1,
$$

where $\mathscr{C}_{M}$ denotes the sheaf of Sato's microfunctions. It microlocalizes the distinguished triangle:

$$
\left.\left.\mathscr{B}_{M}\right|_{N} \longrightarrow \Gamma_{N} \mathscr{B}_{M}\right|_{N}[d] \longrightarrow R \dot{\pi}_{N *} \mu_{N} \mathscr{B}_{M}[d] \longrightarrow+1
$$

considered in Kashiwara-Kawai [6] by setting $\dot{\pi}_{N}: \dot{T}_{N}^{*} M \rightarrow N$, and we believe that the sheaf $\mathscr{C}_{N M}$ is a useful object when studying higher codimensional boundary value problems.

\subsection{Second microfunctions associated to a foliation}

Let $M$ be a real analytic manifold of dimension $n$ and $X$ be its complexification as in the previous section. We assume the manifold $M$ is endowed with an analytic foliation by leaves of dimension $d$ and denote by $L$ the union of their complexification in $X$. Hence, locally there exist a smooth surjective real analytic morphism $g: M \rightarrow M^{\prime}$ and its complexification $g_{\mathbb{C}}: X \rightarrow X^{\prime}$, and $L=g_{\mathbb{C}}^{-1}\left(M^{\prime}\right)$. We shall apply our theory to the triplet $(X, L, M)$. It follows from Theorem 4.3 (ii) and Kashiwara's abstract edge of the wedge theorem that the complex $\mu_{M L}\left(\mathcal{O}_{X}\right)[n]$ on $T_{M}^{*} L \times_{L} T_{L}^{*} X$ is concentrated in degree 0 .

Definition 5.4. We set:

$$
\mathscr{C}_{M L}:=\mu_{M L}\left(\mathcal{O}_{X}\right) \otimes \text { or }_{M}[n]
$$

and call it the sheaf of second microfunctions along $L$.

Now let $\pi_{M}$ denote the projection $T_{M}^{*} L \times{ }_{L} T_{L}^{*} X \rightarrow M \times{ }_{L} T_{L}^{*} X$. Set $\Lambda:=M \times_{L} T_{L}^{*} X \subset T_{M}^{*} X$ and denote by $\pi$ the composition of $\pi_{M}$ and the projection $\Lambda \rightarrow M$. We denote by $\mathscr{C} \mathcal{O}$ the sheaf of microfunctions with holomorphic parameters defined by:

$$
\mathscr{C} \mathcal{O}:=\mu_{L}\left(\mathcal{O}_{X}\right) \otimes \text { or }_{L}[n-d]
$$

Applying Sato's distinguished triangle $R \pi_{M !} \rightarrow R \pi_{M *} \rightarrow R \dot{\pi}_{M *} \rightarrow+1$ to the sheaf $\mathscr{C}_{M L}$, we get:

Theorem 5.5. There exists an exact sequence on $\Lambda=M \times{ }_{L} T_{L}^{*} X$ : 


$$
\left.\left.0 \longrightarrow \mathscr{C O O}\right|_{A} \longrightarrow \mathscr{C}_{M}\right|_{\Lambda} \longrightarrow \dot{\pi}_{M *} \mathscr{C}_{M L} \longrightarrow 0
$$

Hence we have functorially constructed a sheaf which decomposes the singularities of the microfunctions on $\Lambda$. Notice that the sheaf of second microfunctions of Kashiwara [8], that is $\mu_{\Lambda}\left(\mu_{L}\left(\mathcal{O}_{X}\right)\right)[n]$ is different from ours. Also notice that the same sheaf as $\mathscr{C}_{M L}$ was already obtained by Kataoka-Tose [13] making use of their second comonoidal transformation. Refer also to the paper of Kataoka-Tose-Okada [14]. However these authors did not construct it including the zero sections and their formulation was not functorial. In particular, it does not allow them to make use of algebraic machinery of $\mathscr{D}_{X}$-modules. An attempt to extend our construction to the third microlocalization was recently made by Watanabe [26].

Definition 5.6. We denote by $\mathrm{sp}_{L}$ the natural morphisms:

$$
\mathscr{B}_{M} \stackrel{\sim}{\longrightarrow} \pi_{*} \mathscr{C}_{M L},\left.\mathscr{C}_{M}\right|_{\Lambda} \stackrel{\sim}{\longrightarrow} \pi_{M *} \mathscr{C}_{M L} .
$$

If $u$ is a hyperfunction on $M$ (resp. a microfunction on $A$ ), we denote by $\mathrm{ss}_{L}(u)$ the support of $\operatorname{sp}_{L}(u)$ in $T_{M}^{*} L \times_{L} T_{L}^{*} X$.

From now on, we shall explain how the basic operations on second microfunctions can easily be deduced from the morphisms constructed in Section 4. Let $f:(Y, H, N) \rightarrow(X, L, M)$ be a morphism of triplets of manifolds. Here we assume that $\left.f\right|_{Y}: Y \rightarrow X$ is a complexication of the real analytic morphism $\left.f\right|_{N}: N \rightarrow M, H$ is defined similarly to $L$ and $f(H) \subset L$. Then we have canonical morphisms associated to $f$ :

$$
T_{N}^{*} H \times{ }_{H} T_{H}^{*} Y \stackrel{\longleftarrow}{\rho_{f}}\left(N \times{ }_{M} T_{M}^{*} L\right) \times_{L} T_{L}^{*} X \underset{w_{f}}{\longrightarrow} T_{M}^{*} L \times{ }_{L} T_{L}^{*} X .
$$

The following theorem is a direct consequence of Proposition 4.5.

Theorem 5.7. There exist canonical morphisms of "restriction" and "integration" of second microfunctions:

$$
\left\{\begin{array}{l}
\rho_{f_{!}} \varpi_{f}^{-1} \mathscr{C}_{M L} \longrightarrow \mathscr{C}_{N H} \text { and } \\
\varpi_{f_{!}} \rho_{f}^{-1}\left(\mathscr{C}_{N H} \otimes_{\mathscr{A}_{N}} \mathscr{V}_{N}\right) \longrightarrow \mathscr{C}_{M L} \otimes_{\mathscr{A}_{M}} \mathscr{V}_{M},
\end{array}\right.
$$

where $\mathscr{A}$ (resp. $\mathscr{V}$ ) denotes the sheaf of analytic functions (resp. analytic densities) on a real analytic manifold.

For example, assume $N$ is a submanifold of $M$ which is transversal to the leaves of the foliation of $M, Y$ a complexification of $N$ in $X$ and $H=Y \cap L$. Set $\Lambda^{\prime}:=N \times_{H} T_{H}^{*} Y$. Then $\Lambda^{\prime} \simeq N \times_{M} \Lambda$ and we have canonical morphisms:

$$
T_{N}^{*} H \times{ }_{N} \Lambda^{\prime} \longleftarrow\left(N \times{ }_{M} T_{M}^{*} L\right) \times{ }_{M} \Lambda \underset{\sigma}{\longrightarrow} T_{M}^{*} L \times{ }_{M} \Lambda,
$$


where $\rho$ is smooth and $\varpi$ is a closed embedding. Hence we find that if $\left.u \in \mathscr{C}_{M}\right|_{\Lambda}$ and $\operatorname{ss}_{L}(u)$ is proper with respect to $\rho$, then $\left.\left.u\right|_{N} \in \mathscr{C}_{N}\right|_{\Lambda^{\prime}}$ is well-defined. It implies the possibility of defining a local restriction of microfunctions under a suitable condition on the second singular spectrum. It seems to be a new fact, because until now the restriction of microfunctions were always considered to be global.

Remark 5.8. The operations in Theorem 5.7 generalizes those obtained by Okada-Tose [17] for compactly supported hyperfunctions in a non functorial way. They defined them by means of the FBI transformation of [23]. Our construction ensures that these operations are coordinate invariant and well-defined for microfunctions.

Finally let us consider the "product" of second microfunctions. Let $(X, L, M)$ be the triplet as before and take a coordinate system $\left(x, \sqrt{-1} \eta^{\prime} d x^{\prime}\right.$, $\left.\sqrt{-1} \eta^{\prime \prime} d x^{\prime \prime}\right)$ of $T_{M}^{*} L \times_{L} T_{L}^{*} X$ in which $\left(x, \sqrt{-1} \eta^{\prime} d x^{\prime}\right)$ belongs to $T_{M}^{*} L$. In this case, the morphism $\theta$ in (4.38) is the morphism:

$$
\left(T_{M}^{*} L \times{ }_{L} T_{L}^{*} X\right) \times{ }_{M}\left(T_{M}^{*} L \times{ }_{L} T_{L}^{*} X\right) \longrightarrow T_{M}^{*} L \times{ }_{L} T_{L}^{*} X
$$

which is described by

$$
\begin{aligned}
& \left(\left(x, \sqrt{-1} \eta_{1}^{\prime} d x^{\prime}, \sqrt{-1} \eta_{1}^{\prime \prime} d x^{\prime \prime}\right),\left(x, \sqrt{-1} \eta_{2}^{\prime} d x^{\prime}, \sqrt{-1} \eta_{2}^{\prime \prime} d x^{\prime \prime}\right)\right) \\
\longmapsto & \left(x, \sqrt{-1}\left(\eta_{1}^{\prime}+\eta_{2}^{\prime}\right) d x^{\prime}, \sqrt{-1}\left(\eta_{1}^{\prime \prime}+\eta_{2}^{\prime \prime}\right) d x^{\prime \prime}\right) .
\end{aligned}
$$

Here we sometimes consider $M$ as a submanifold of $T_{M}^{*} L \times_{L} T_{L}^{*} X$ by identifying it with the intersection of two zero sections, that is, $T_{M}^{*} L \cap\left(M \times_{L} T_{L}^{*} X\right)$. In general, we cannot make a product of two hyperfunctions $u_{1}$ and $u_{2}$. In order to give a criterion which assures the possibility of the product operation, we prepare the next lemma.

Lemma 5.9. Let $S$ be a closed subset of $\left(T_{M}^{*} L \times{ }_{L} T_{L}^{*} X\right) \times{ }_{M}\left(T_{M}^{*} L \times{ }_{L} T_{L}^{*} X\right)$ which is conic with respect to the four conic structures. Then the following two conditions are equivalent each other.

(i) $\theta$ is a proper mapping over $S$.

(ii) $\theta$ satisfies:

$$
\left\{\begin{array}{l}
S \cap \theta^{-1}\left(T_{M}^{*} L\right) \subset\left(T_{M}^{*} L\right) \times{ }_{M}\left(T_{M}^{*} L\right) \quad \text { and } \\
S \cap \theta^{-1}\left(M \times{ }_{L} T_{L}^{*} X\right) \subset\left(M \times{ }_{L} T_{L}^{*} X\right) \times{ }_{M}\left(M \times{ }_{L} T_{L}^{*} X\right) .
\end{array}\right.
$$

Proof. It follows from the condition (ii) that:

$$
S \cap \theta^{-1}(M) \subset M \times{ }_{M} M \simeq M .
$$

Hence $\theta$ is proper over $S$, because $\theta$ is a linear mapping w.r.t. the fibers. Next 
assume the condition (i) and for example take a point:

$$
p_{0}:=\left(\left(x, \sqrt{-1} \eta_{1}^{\prime} d x^{\prime}, \sqrt{-1} \eta_{1}^{\prime \prime} d x^{\prime \prime}\right),\left(x, \sqrt{-1} \eta_{2}^{\prime} d x^{\prime}, \sqrt{-1} \eta_{2}^{\prime \prime} d x^{\prime \prime}\right)\right)
$$

in $S \cap \theta^{-1}\left(M \times{ }_{L} T_{L}^{*} X\right)$, that is, $\eta_{1}^{\prime}+\eta_{2}^{\prime}=0$. Suppose $p_{0}$ does not belong to $\left(M \times{ }_{L} T_{L}^{*} X\right) \times{ }_{M}\left(M \times{ }_{L} T_{L}^{*} X\right)$, then $\eta_{1}^{\prime}=-\eta_{2}^{\prime} \neq 0$. Since $S$ is conic w.r.t. $\eta_{1}^{\prime \prime}$ and $\eta_{2}^{\prime \prime}$ variables, we get a point:

$$
q_{0}:=\left(\left(x, \sqrt{-1} \eta_{1}^{\prime} d x^{\prime}, \sqrt{-1} 0 d x^{\prime \prime}\right),\left(x, \sqrt{-1} \eta_{2}^{\prime} d x^{\prime}, \sqrt{-1} 0 d x^{\prime \prime}\right)\right)
$$

which belongs to $\left[S \cap \theta^{-1}(M)\right] \backslash\left[M \times{ }_{M} M\right]$. It contradicts the assumption (i).

Now we can give a new condition which ensures the existence of the product of two hyperfunctions.

Theorem 5.10. Let $u_{1}$ and $u_{2}$ be hyperfunctions on M. Assume every point:

$$
\left(\left(x, \sqrt{-1} \eta_{1}^{\prime} d x^{\prime}, \sqrt{-1} \eta_{1}^{\prime \prime} d x^{\prime \prime}\right),\left(x, \sqrt{-1} \eta_{2}^{\prime} d x^{\prime}, \sqrt{-1} \eta_{2}^{\prime \prime} d x^{\prime \prime}\right)\right)
$$

in $\mathrm{ss}_{L}\left(u_{1}\right) \times{ }_{M} \mathrm{ss}_{L}\left(u_{2}\right)$ satisfies the following conditions:

$$
\left\{\begin{array}{l}
\eta_{1}^{\prime}+\eta_{2}^{\prime}=0 \Longrightarrow \eta_{1}^{\prime}=\eta_{2}^{\prime}=0 \quad \text { and } \\
\eta_{1}^{\prime \prime}+\eta_{2}^{\prime \prime}=0 \Longrightarrow \eta_{1}^{\prime \prime}=\eta_{2}^{\prime \prime}=0 .
\end{array}\right.
$$

Then there exists a "product" $u_{1} \cdot u_{2}$ of $u_{1}$ and $u_{2}$, and

$$
\operatorname{ss}_{L}\left(u_{1} \cdot u_{2}\right) \subset \theta\left[\operatorname{ss}_{L}\left(u_{1}\right) \times{ }_{M} \operatorname{ss}_{L}\left(u_{2}\right)\right] .
$$

Proof. It is an immediate consequence of Proposition 4.7 and Lemma 5.9.

\subsection{Second microdifferential operators}

In this section, we shall explain some ideas in order to develop the theory of second microdifferential operators which act on our microfunctions. Notice that the class of operators of Laurent [15] seems to be too wide to operate on the sheaf $\mathscr{C}_{M L}$. First, let $(X, Y, Z)$ be a triplet of complex manifolds and assume $Z$ is of complex codimension $d$ in $X$. Then we will show the result below in a forthcoming paper.

Proposition 5.11. The complex $\mu_{Z Y}\left(\mathcal{O}_{X}\right)[d]$ of sheaves on $T_{Z}^{*} Y \times_{Y} T_{Y}^{*} X$ is concentrated in degree 0 , and we define the sheaf $\mathscr{C}_{Z Y \mid X}^{\mathbb{R}}$ of second holomorphic microfunctions by:

$$
\mathscr{C}_{Z Y \mid X}^{\mathbb{R}}:=\mu_{Z Y}\left(\mathcal{O}_{X}\right)[d] .
$$

It is possible to write down the various functorial properties of second holomorphic microfunctions as in [19], but we shall not develop it here. Let 
$X=X^{\prime} \times X^{\prime \prime}$ be a product of two complex manifolds. We denote by $\Delta_{X}=\Delta_{X^{\prime}} \times \Delta_{X^{\prime \prime}}$ the diagonal set of $X \times X$ and set $\hat{L}:=X^{\prime} \times X^{\prime} \times \Delta_{X^{\prime \prime}}$ in $X \times X$. Let us apply our functor to the triplet $\left(X \times X, \hat{L}, \Delta_{X}\right)$ of complex manifolds.

Definition 5.12. We define the sheaf $\mathscr{E}_{X L}^{\mathbb{R}}$ of second microdifferential operators on

$$
T_{\Delta x}^{*} \hat{L} \times \hat{L} T_{\hat{L}}^{*}(X \times X) \simeq T^{*} X^{\prime} \times T^{*} X^{\prime \prime}
$$

by $\mathscr{E}_{X L}^{\mathbb{R}}:=\mu_{\Delta_{X} \hat{L}}\left(\mathcal{O}_{X \times X}^{(0, n)}\right)[n]$, where $n=\operatorname{dim} \mathbb{} X$ and $\mathcal{O}_{X \times X}^{(0, n)}$ denotes the sheaf of holomorphic differential forms of maximal degree w.r.t. the second factor.

We can easily verify that the sheaf $\mathscr{E}_{X L}^{\mathbb{R}}$ is endowed with a ring structure by the results of Kashiwara-Schapira [9]. Moreover the restriction of the sheaf $\mathscr{E}_{X L}^{\mathbb{R}}$ to the zero section

$$
\Delta_{X} \times \hat{L}_{\hat{L}} T_{\hat{L}}^{*}(X \times X) \simeq X^{\prime} \times T^{*} X^{\prime \prime}
$$

coincides with the restriction of the sheaf $\mathscr{E}_{X}^{\mathbb{R}}$ of holomorphic microdifferential operators to it. This is an explicit advantage of our theory. Now let $M=M^{\prime} \times M^{\prime \prime}$ be a product of two real analytic manifolds, $X=X^{\prime} \times X^{\prime \prime}$ a complexification and $L:=X^{\prime} \times M^{\prime \prime}$ a partial complexification of $M=M^{\prime} \times M^{\prime \prime}$ in $X$. We shall consider the action of the Ring $\mathscr{E}_{X L}^{\mathbb{R}}$ on the sheaf $\mathscr{C}_{M L}$ of second microfunctions along $L$. For this purpose, we perform a so-called S-K-K realification. To begin with, define the sheaf $\mathscr{C}_{(M \times M)(L \times L)}$ of second microfunctions on

$$
T_{(M \times M)}^{*}(L \times L) \underset{(L \times L)}{\times} T_{(L \times L)}^{*}(X \times X) \simeq T^{*}\left(M^{\prime} \times M^{\prime}\right) \times T^{*}\left(M^{\prime \prime} \times M^{\prime \prime}\right)
$$

by:

$$
\mathscr{C}_{(M \times M)(L \times L)}:=\mu_{(M \times M)(L \times L)}\left(\mathcal{O}_{X \times X}\right) \otimes \text { or }_{M \times M}[2 n] .
$$

Then we have:

Theorem 5.13. There exists a canonical morphism of "realification":

$$
\left.\mathscr{E}_{X L}^{\mathbb{R}}\right|_{T_{M}^{*}, X^{\prime} \times T_{M^{\prime \prime}}^{*} X^{\prime \prime}} \longrightarrow \Gamma_{E} \mathscr{C}_{(M \times M)(L \times L)} \otimes_{\mathscr{A}_{M}} \mathscr{V}_{M},
$$

where we set:

$$
\begin{aligned}
E & :=T_{\Delta_{M^{\prime}}}^{*}\left(M^{\prime} \times M^{\prime}\right) \times T_{\Delta_{M^{\prime \prime}}}^{*}\left(M^{\prime \prime} \times M^{\prime \prime}\right) \\
& \simeq T^{*} M^{\prime} \times T^{*} M^{\prime \prime} \simeq T_{M^{\prime}}^{*} X^{\prime} \times T_{M^{\prime \prime}}^{*} X^{\prime \prime}
\end{aligned}
$$

Proof. The intersection of the triplets $\left(X \times X, \hat{L}, \Delta_{X}\right)$ and $(X \times X, L \times L$, $M \times M)$ is equal to 


$$
\left(X \times X, \hat{L} \cap(L \times L), \Delta_{X} \cap(M \times M)\right)=\left(X \times X, L^{\prime}, \Delta_{M}\right)
$$

by setting $L^{\prime}:=X^{\prime} \times X^{\prime} \times \Delta_{M^{\prime \prime}}$. Therefore there exist morphisms of triplets of manifolds:

$$
\left\{\begin{array}{l}
f:=\operatorname{id}_{X \times X}:\left(X \times X, L^{\prime}, \Delta_{M}\right) \longrightarrow\left(X \times X, \hat{L}, \Delta_{X}\right), \\
g:=\operatorname{id}_{X \times X}:\left(X \times X, L^{\prime}, \Delta_{M}\right) \longrightarrow(X \times X, L \times L, M \times M) .
\end{array}\right.
$$

By virtue of the results in Section 4.2, we have associated injective morphisms:

$$
\begin{aligned}
T_{\Delta_{M}}^{*} L^{\prime} \times{ }_{L^{\prime}} T_{L^{\prime}}^{*}(X \times X) & \underset{\rho_{f}}{\stackrel{\Delta_{M}}{\times}} \Delta_{\Delta_{X}}\left[T_{\Delta_{X}}^{*} \hat{L} \times \hat{L} T_{\hat{L}}^{*}(X \times X)\right] \\
& \underset{\varpi_{f}}{\longrightarrow} T_{\Delta_{X}}^{*} \hat{L} \times \hat{L} T_{\hat{L}}^{*}(X \times X)
\end{aligned}
$$

and

$$
\begin{aligned}
T_{\Delta_{M}}^{*} L^{\prime} \times L_{L^{\prime}} T_{L^{\prime}}^{*}(X \times X) \underset{\rho_{g}}{\stackrel{\leftarrow}{\Delta_{M}}} \Delta_{M} \underset{(M \times M)}{\times}\left[T_{(M \times M)}^{*}(L \times L) \underset{(L \times L)}{\times} T_{(L \times L)}^{*}(X \times X)\right] \\
\underset{\sigma_{g}}{\longrightarrow} T_{(M \times M)}^{*}(L \times L) \underset{(L \times L)}{\times} T_{(L \times L)}^{*}(X \times X) .
\end{aligned}
$$

The next lemma is a simple corollary of Proposition 4.5.

Lemma 5.14. Let $F \in \mathbb{D}^{\mathrm{b}}(X \times X)$. Then there exist canonical morphisms:

$$
\left\{\begin{array}{l}
\varpi_{f}^{-1} \mu_{\Delta_{X} \hat{L}}(F) \longrightarrow \rho_{f}^{!} \mu_{\Delta_{M} L^{\prime}}(F) \otimes o r_{M}[n], \\
\rho_{g}^{-1} \mu_{\Delta_{M} L^{\prime}}(F) \longrightarrow \varpi_{g}^{!} \mu_{(M \times M)(L \times L)}(F) .
\end{array}\right.
$$

Now let us set:

$$
\begin{aligned}
E & :=\Delta_{M_{\Delta X}}^{\times}\left[T_{\Delta_{X}}^{*} \hat{L} \times \hat{L}_{\hat{L}} T_{\hat{L}}^{*}(X \times X)\right] \\
& \cap \Delta_{\left.M_{(M} \times M\right)}\left[T_{(M \times M)}^{*}(L \times L) \underset{(L \times L)}{\times} T_{(L \times L)}^{*}(X \times X)\right] \\
& \simeq T_{\Delta_{M^{\prime}}}^{*}\left(M^{\prime} \times M^{\prime}\right) \times T_{\Delta_{M^{\prime \prime}}}^{*}\left(M^{\prime \prime} \times M^{\prime \prime}\right)
\end{aligned}
$$

in $T_{\Delta_{M}}^{*} L^{\prime} \times{ }_{L^{\prime}} T_{L^{\prime}}^{*}(X \times X)$, and consider the Cartesian square below:

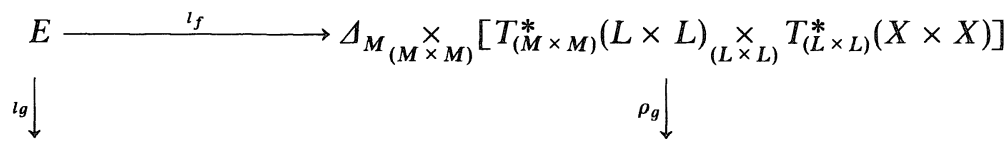

$$
\Delta_{M} \underset{\Delta_{X}}{\times}\left[T_{\Delta_{X}}^{*} \hat{L} \times \hat{L}_{\hat{L}} T_{\hat{L}}^{*}(X \times X)\right] \longrightarrow T_{\Delta_{M}}^{*} L^{\prime} \times_{L^{\prime}} T_{L^{\prime}}^{*}(X \times X)
$$

in which all morphisms are injective. Therefore we get a chain of morphisms: 


$$
\begin{aligned}
\left.\mu_{\Delta_{X} \hat{L}}\left(\mathcal{O}_{X \times X}\right)\right|_{E}[n] & =l_{g}^{-1} \varpi_{f}^{-1} \mu_{\Delta_{X} \hat{L}}\left(\mathcal{O}_{X \times X}\right)[n] \\
& \rightarrow l_{g}^{-1} \rho_{f}^{\vdots} \mu_{\Delta_{M} L^{\prime}}\left(\mathcal{O}_{X \times X}\right) \otimes \operatorname{or}_{M}[2 n] \\
& \rightarrow l_{f} \rho_{g}^{-1} \mu_{\Delta_{M} L^{\prime}}\left(\mathcal{O}_{X \times X}\right) \otimes \operatorname{or}_{M}[2 n] \\
& \rightarrow l_{f} \varpi_{g}^{!} \mu_{(M \times M)(L \times L)}\left(\mathcal{O}_{X \times X}\right) \otimes \operatorname{or}_{M}[2 n] \\
& \simeq R \Gamma_{E} \mu_{(M \times M)(L \times L)}\left(\mathcal{O}_{X \times X}\right) \otimes \operatorname{or}_{M}[2 n]
\end{aligned}
$$

due to the above lemma, and the proof of the theorem is complete.

Theorem 5.13 asserts that our second microdifferential operators are transformed to second microlocal operators, i.e. elements of $\Gamma_{E} \mathscr{C}_{(M \times M)(L \times L)}$ $\otimes_{\mathscr{A}_{M}} \mathscr{V}_{M}$. The action of second microlocal operators on the sheaf $\mathscr{C}_{M L}$ can be easily obtained by making use of Theorem 5.7 and Theorem 5.10 along the same lines as in Sato-Kawai-Kashiwara [19], and we shall omit precise arguments here.

\section{References}

[1] Bost, J-B., Deformation to the normal cone and index theorem, Exposé au congrès satellite ECM, Université Paris Nord, 30 juin, 1992.

[2] Delort, J-M., Microlocalisation simultanée et problème de Cauchy ramifié, Compositio Math., to appear.

[3] Ishimura, R., Le foncteur $\mu$ hom relatif et l'action de $\mathscr{E}^{\mathbb{R}}$ relatif sur les fonctions holomorphes, Mem. Fac. Sci. Kyushu Univ., Ser A46 (1992), 69-77.

[4] Kashiwara, M., Deuxième microlocalisation et théorème du "watermelon", Talks at Nice, unpublished, 1972.

[5] - Systems of microdifferential equations, Progr. Math. 34, Birkhäuser, Boston, 1983.

[6] Kashiwara, M. and Kawai, T., On the boundary value problem for elliptic systems of linear partial differential equations I-II, Proc. Japan. Acad., 48 (1971), 712-715; ibid., 49 (1972), $164-168$.

[7] - Second microlocalization and asymptotic expansions, LN in Physics 126, Proc. Les Houches, D. Iagolnitzer editor, Springer-Verlag, 79 (1979), 21-76.

[8] Kashiwara, M. and Laurent, Y., Théorèmes d'annulation et deuxième microlocalisation, prépublication d'Orsay, 1983.

[9] Kashiwara, M. and Schapira, P., Micro-hyperbolic systems, Acta Math., 142 (1979), 1-55.

[10] - Microlocal study of sheaves, Astérisque, 128 (1985).

[11] — Sheaves on manifolds, Grundlehien der Math. Wiss. 292, Springer-Verlag, 1990.

[12] Kataoka, K., On the theory of Radon transformations of hyperfunctions, J. Fac. Sci. Univ. Tokyo, 28 (1981), 331-413.

[13] Kataoka, K. and Tose, N., Some remarks in 2nd microlocalization (in Japanese), RIMS Kôkyu-roku, Kyoto Univ., 660 (1988), 52-63.

[14] Kataoka, K., Tose, N. and Okada, Y., Decomposition of second microlocal analytic singularities, D-Modules and Microlocal Geometry, M. Kashiwara, T. Monteiro-Fernandès and P. Schapira editors., De Gruyter, (1993), 163-171.

[15] Laurent, Y., Théorie de la deuxième microlocalisation dans le domaine complexe, Progress in Math., Birkhaüser, 1985. 
[16] Oaku, T., Higher-codimensional boundary value problem and $F$-mild hyperfunctions, Algebraic Analysis, II, (Papers dedicated to Prof. Sato), M. Kashiwara and T. Kawai editors., Academic Press, (1988), 571-586.

[17] Okada, Y. and Tose, N., FBI-transformation and microlocalization-equivalence of the second analytic wave front sets and the second singular spectrum, J. Math. Pures Appl., 70.4 (1991), 427-455.

[18] Sabbah, C., Quelque remarques sur la geometrie des cones conormaux, Astérisque, 130 (1985), 161-192.

[19] Sato, M., Kawai, T. and Kashiwara, M., Hyperfunctions and pseudodifferential equations, $L N$ in Math, Springer-Verlag, 287 (1973), 265-529.

[20] Schapira, P., Microfunctions for boundary value problems, Algebraic Analysis, II, (Papers dedicated to Prof. Sato), M. Kashiwara and T. Kawai editors., Academic Press, (1988) 809-819.

[21] Schapira, P. and Schneiders, J-P., Index theorem for elliptic pairs, Astérisque, 224 (1994).

[22] Schapira, P. and Takeuchi, K., Déformation binormale et bispécialisation, C.R. Acad. Sci., 319, Série I (1994), 707-712.

[23] Sjöstrand, J., Singularités analytiques microlocales, Astérisque, 95 (1982).

[24] Takeuchi, K., Étude microlocale des problèmes aux limites en codimension supérieure, C.R. Acad. Sci., 320, Série I (1995), 441-443.

[25] - Microlocal boundary value problem in higher codimensions, to appear in Bull. Soc. Math. France.

[26] Watanabe, S., On third microlocal analysis, Master thesis presented to University of Chiba., 1995. 